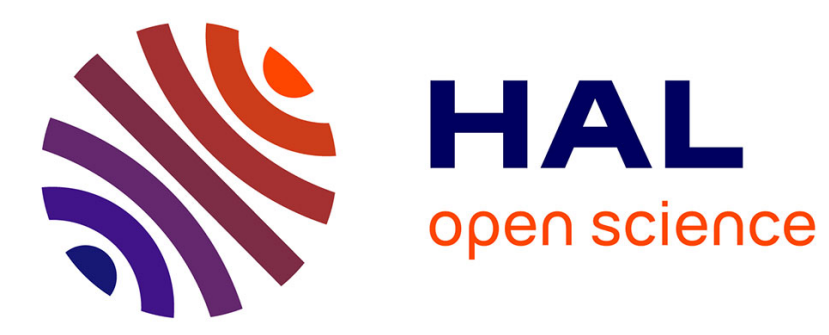

\title{
Contribution of computational mechanics in numerical simulation of machining and blanking: State-of-the-Art
}

Philippe Lorong, Julien Yvonnet, Gérard Coffignal, Stéphanie

Assouline-Cohen

\section{- To cite this version:}

Philippe Lorong, Julien Yvonnet, Gérard Coffignal, Stéphanie Assouline-Cohen. Contribution of computational mechanics in numerical simulation of machining and blanking: State-of-the-Art. Archives of Computational Methods in Engineering, 2006, 13 (1), pp.45-90. 10.1007/BF02905931 . hal-00019220

\section{HAL Id: hal-00019220 \\ https://hal.science/hal-00019220}

Submitted on 17 Apr 2018

HAL is a multi-disciplinary open access archive for the deposit and dissemination of scientific research documents, whether they are published or not. The documents may come from teaching and research institutions in France or abroad, or from public or private research centers.
L'archive ouverte pluridisciplinaire HAL, est destinée au dépôt et à la diffusion de documents scientifiques de niveau recherche, publiés ou non, émanant des établissements d'enseignement et de recherche français ou étrangers, des laboratoires publics ou privés. 


\title{
Contribution of Computational Mechanics in Numerical Simulation of Machining and Blanking: State-of-the-Art
}

\author{
$\mathrm{Ph}$. Lorong, J. Yvonnet, G. Coffignal and S. Cohen \\ LMSP: Laboratory for Mechanics of Systems and Processes \\ ENSAM Paris \\ 151 Boulevard de l'Hôpital, 75013 Paris, France \\ philippe.lorong@paris.ensam.fr
}

\section{Summary}

Blanking and machining are commonly used in processes to obtain the shape of many mechanical pieces. Although considerable number of experimental results exist, certain essential aspects of cutting are still not well understood. This comes from the complexity of the thermomechanical phenomena induced by the material separation as well as from the complexity of the dynamical behaviour of the whole workpiece/tool/machine system. Numerical simulations make it possible to go further in the comprehension and the prediction of machining and cutting processes.

In this work the state-of-the art is analysed and we present the most recent developments in the contribution of computational mechanics to numerical simulation of machining and blanking. This contribution is, on one hand, developed at a very global scale called macroscopic scale. At this scale a representation of the deformations of the piece is necessary, for example when thin walls are present, and when both predictions of the geometrical state of final surface and/or stability of the process are expected. On the other hand, the contribution is also located at a more local scale: the mesoscopic scale. At this scale, the aim is the determination of thermomechanical sollicitations applied to the tool, the simulation of chip formation, or the description of residual states (mechanical, chemical) inside the workpiece after machining.

\section{INTRODUCTION}

During machining, metallurgical and chemical transformations, as well as dynamical phenomena produced by the cutting process, occur in the cutting area. Their analysis, including phase changes and chemical diffusion between chip and tool, will bring out important features on material flow during cutting and on cutting capability for a given material.

Whenever understanding or optimisation of cutting conditions is studied, it is highly important to have numerical tools that can simulate the operations. For example in the field of metal forming, some efficient simulation codes have been developed during the last decade for stamping and sheet forming. They are now widely used in industry.

In metal cutting, except for some specific application (CUTPRO [1] for the stability of the process, AdvantEdge ${ }^{T M}$ [2] for machining) we do not dispose of such tools. This is due to the complexity of the forming process wich involves high dynamical shear and friction mechanisms, inducing heat generation.

Indeed, the thermomechanical coupling which occurs involves viscoplasticity at large strains (up to 10) and large stain rates (up to $10^{5} s^{-1}$ ) where the material goes from room temperature to the heated state in few milliseconds, heat coming from internal dissipation and friction. In addition, the constant evolution of the workpiece - due to material removal - and the complexity of the dynamic behaviour of the machine, particularly for high speed spindles, are leading to additional difficulties. Finally it is noteworthy that the increase of the cutting speeds increases the role of the thermal phenomena and thermomechanic coupling, by causing the localization of the strain and the creation of shear bands - called adiabatic shear bands - that have a very thin thickness (between $5.10^{-5} \mathrm{~m}$ and $10^{-5} \mathrm{~m}$ ). 
It is a common habit in computational mechanics, as in physical science, to define a simulation model which is only a representation model of reality. The assumptions done to build on the model depend on the nature of the expected results. For example, to make a modal analysis a coarse mesh may be sufficient, but to make a stress analysis a fine mesh must be used in the area of interest. It is clear that all the above phenomena observed during the cutting process need appropriate reference scales to be properly and consistently analysed: the separation of physical effect with large length of variation from those with smaller characteristic length must be done. We shall hereafter introduce three different scales (see Figure 1) to consistently study the cutting operation [3]:

- a microscopic scale at the material grain level. This scale will be required to analyse the material microstucture; for example to construct a polycrystalline thermomechanical constitutive law including grain distribution or to take into account phase changes, chemical diffusion, damage, wear, ...

- a mesoscopic scale at the tool tip/workpiece material level the material is modeled as a continuum domain. In blanking the mesoscopic scale is used to study the whole material separation front inside the workpiece. In machining, this scale is the usual one to make the study of continuous chip formation, in order to predict chip geometry, stresses and temperatures, as well as the repartition of the cutting forces along the tool-tip, the thermomechanical sollicitations on the tool-tip (determination of toolchip and tool-workpiece frictions).

- a macroscopic scale to analyse the whole workpiece-tool-machine level. This macroscopic scale will be more appropriate when dealing with dynamics of the system. At this scale the tool is simply seen as an erasing tool.

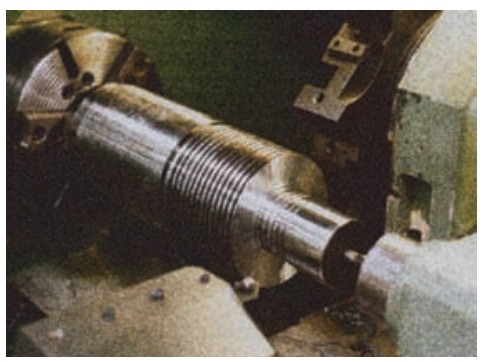

Macroscopic scale

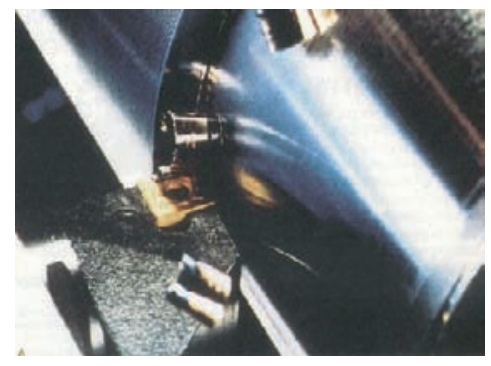

Mesoscopic scale

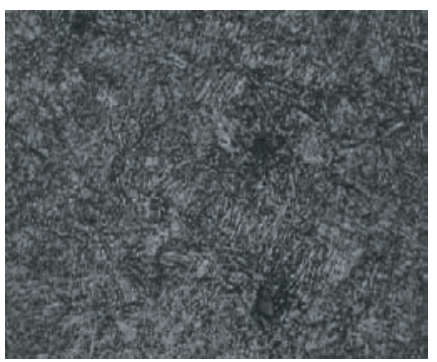

Microscopic scale

Figure 1. The multiscale partition

Computational mechanics is an essential component of the tools that are avaliable to make it possible to obtain, in a short time, operational results. It takes place mainly in both macroscopic and mesoscopic scales in the following manner:

- at the macroscopic scale it is an usual way to model the dynamic behaviour of the machine. For pieces including deep pockets or thin walls, a more specific use of computational mechanics is encountered as it is necessary to take into account the deformations of the piece inside the machined zone.

- at the mesoscopic scale to compute complex and time dependent space fields. For example it permits to make an accurate analysis of stresses and temperatures. 
The two next sections are concerning simulation of machining at the macroscopic scale. Section 2 recalls the goals and context, gives an overview of the current approaches, and specifies different ways of using computational mechanics at this scale. Section 3 focuses on the case of flexible workpieces such as thin-walled workpieces. An accurate definition, and practical aspects, of material removal are given in this context. Sections 4 and 5 are dealing with the simulation at the mesoscopic scale. Section 4 presents an overview of simulation at this scale and points out some remaining difficulties. In section 5 we propose an alternative to finite element approaches, the C-NEM, to break up the non-reliability of 3D re-meshing. Some results will be presented in the case of blanking.

\section{NUMERICAL SIMULATION AT THE MACROSCOPIC SCALE}

\subsection{Goals and Context of Simulations at the Macroscopic Scale}

The main goals of simulations at the macroscopic scale are to predict the vibrational behaviour during machining (displacements, cutting forces), including vibrational instabilities such as chatter, and possibly the resulting final surface (surface finish, roughness and detailed description of its geometry). Sufficiently accurate mechanical and geometric models have to be built in order to reproduce with fidelity the vibrational behaviour of the system.

An important aspect of the simulation at the macroscopic level is the nature of the tool/workpiece interaction which is history dependent. This is due to the evolution, during machining, of the workpiece domain and boundary. To follow this evolution, the material removal is modeled via the concept of an erasing tool (wherever the tool passes the corresponding workpiece material disappears) associated to a cutting law which gives the resulting forces versus, among others, the cutting section, which is time dependent and previously qualified of history dependent $[4,5]$. As the cutting section encountered by the tip of one tool is dependent of previous passing of itself or other tool tip, self-excited machine behaviour can appear and may conduct to chatter. One of the first theory of self-excited machine tool chatter was proposed in 1965 by Merritt in [6].

The dynamic model includes the machine, the tool and the workpiece. It can be any type of model giving a good description of the real physical behaviour (from a simple massspring model to a complex finite element model for instance). This mechanical model may include the spindle, bearings, some machine parts and workpiece if its flexibility cannot be neglected. The dynamic model can also be subjected to evolutions. For instance, large motions of some parts of the machine affect directly stiffness and mass repartitions. Moreover, in the case of a thin walled piece, the evolution of thickness during machining leads to important reduction of bending stiffness. All these evolutions are quite predictable and simulations, where dynamic characteristics can be taken as piecewise constant, can be done.

The following models are used at the macroscopic scale:

1. Mechanical models for the dynamics of the workpiece, the machine and the tool.

2. Geometry models of the tool, and also of the workpiece to enable its surface (its boundary) to be known anytime (when the workpiece is flexible, its vibrational deformation must be deduced from the mechanical model).

3. A cutting law which gives the resulting forces versus, among others, the cutting section.

As large motions are known (spindle rotation in milling, workpiece rotation in turning, translational displacement of tables and carriages), only small variation of them have to be calculated. Thus an infinitesimal transformation frame can be chosen to conduct the analysis at the macroscopic scale. 


\subsection{Overview of the Current Approaches}

Mainly two approaches aim to predict machining stability at the macroscopic scale. The first one is analytical and can be identified as a periodic approach. Its main idea is to study only machining stability assuming that the process is periodic and that the small variations which occur only cause small consequences. The second approach, identified as a time domain approach, is based on a time study (incremental solving process), which has a rather high numerical cost. The complete history of the evolution of the cutting part of the tool as well as of the workpiece surface is simulated.

In the periodic approach a linearized model is build and its theoritical stability is studied $[7,8,9,10,11,12,13]$. The periodic approach leads to stability lobes which give the areas where cutting is stable as a function of the rotation speed and the depth of cut. The representation of the machined surface is generally very simple: only the passage of the preceding tooth is taken into account to determine the volume of material removed by a tooth. Moreover the piece is supposed perfectly rigid in the machined zone. Several software are used in the industry $[14,15,16,17]$ and results are good as long as the assumptions are true. The limits of these assumptions are often encountered during finishing operations where even small vibrations induce a high variation of the cutting forces.

In the time domain approach $[19,20,21,22,23,13]$, the updating of the workpiece surface, which must be done at each increment, needs a very robust geometric algorithm and takes the major part of the numerical time. Different kinds of geometry models can be used for the workpiece. For example in 3D the following representations are used: Dexels [24, 25, 26], Voxels [27], triple-nailboards models[28], boundary representations (B-Rep) based on triangular tessellation [29], or layer wise representations $[30,31,19,32]$. The major interest of the time domain approach is its ability to simulate any type of cutting conditions. But the definition of a stability criteria is not immediate as it cannot be easily linked to an analytical one. Generally the evaluation of the quality of the surface generated or the level of vibrations of certain parts of the system are used. An example of such a stability criteria is propose by K. Mehdi in [33]. The time domain approach admits the introduction of a flexible workpiece, even in the region where the machining is done.

\subsection{Specific Use of Computational Mechanics at the Macroscopic Scale}

As in other applications, a finite element approach may be used in machining to construct parts of the dynamic model. For example beam based model can be used for the spindle or for the body of the tool if it has a long overhang. As usual, a compromise must be done between quality of representation, complexity of the model and amount of time spent in calculation.

Generally the workpiece undergoes deformations due to clamping, cutting forces, thermal expansion. However, two categories of cases must be distinguished: the case of a workpiece that can be considered as rigid in the region where the cutting teeth are operating, and the case of a workpiece which undergoes deformations in the machined region. When the machined parts of workpiece are flexible, as it is the case of thin-walled pieces, two specific difficulties occur. The first one is due to the deformation of the machined surface, which is a part of the piece boundary. It is not immediate to define material removal in the context where both tool and workpiece boudary are moving, while the workpiece deforms itself. The second one is due to the material removal which affects signigicantly and continuously mass and stiffness of slenderness parts.

For the first case, if the interaction between tool and workpiece affects a large zone of the piece, the mechanical model must take into account a large area and thus a finite element model is well suitable. The surface model must be constrained to follow the given finite element kinematics. For the second case, it is hardly to envisage to make a continuous 
update of the mesh: the numerical costs would be prohibitive. Thus, alternative approaches must be introduced.

In the next chapter, the general mechanical frame is presented (section 3.1), and then we propose an accurate definition of material removal in the context of flexible workpiece (section 3.2). This definition is compatible with the assumption of an erasing tool. Practical aspects, in particular those linked to the use of a finite element model, are developed in section 3.2.2 and 3.2.3. Before to give some examples in section 3.5 we suggest an efficient technique to take into account the evolution of the mass and stiffness matrices (section 3.3) for thin-walled pieces.

\section{TAKING INTO ACOUNT INSTANTANEOUS WORKPIECE DEFORMATION AT THE MACROSCOPIC SCALE}

\subsection{General Mechanical Frame}

As stated before, in the context of the time domain approach, the mechanical model of the Workpiece-Tool-Machine system used can be a simple mass-spring model or a complex Finite Element one, as long as it gives a good description of the real dynamics. It leads to write the equilibrium equation as:

$$
\underline{\underline{M}} \underline{\ddot{q}}+\underline{\underline{C}}(\omega) \underline{\dot{q}}+\underline{\underline{K}} \underline{q}=\underline{Q}_{c u t}\left(\underline{q}, \underline{\dot{q}}, \partial \Omega_{c u t}\right)
$$

where $\underline{\underline{M}}$ is the mass matrix, $\underline{\underline{C}}(\omega)$ is the damping and Coriolis matrix, $\underline{\underline{K}}$ is the stiffness matrix, $\underline{q}$ represents the column of degrees of freedom, $\underline{Q}_{c u t}\left(\underline{q}, \dot{q}, \Gamma_{c u t}\right)$ is the vector representing the cutting forces.

In the macroscopic approach, the main assumption is based on the concept of an erasing tool: wherever the tool passes, the corresponding workpiece material disappears. But a model must be introduced to determine the internal forces to apply between tool and workpiece in coherence with this erasing vision: the cutting law gives the cutting forces from the chip section associated with $\Gamma_{c u t}$ which depends on time. It then allows to calculate $\underline{Q}_{c u t}$.

$\underline{\underline{M}}, \underline{\underline{C}}$ and $\underline{\underline{K}}$ are considered constant throughout the machining operation, as long as this operation $\overline{\bar{d}}$ oes not significantly modify the mass and stiffness characteristics of the workpiece or machine (see section 3.3).

Equation (1) is non-linear because $\underline{Q}_{c u t}\left(\underline{q}, \underline{\dot{q}}, \Gamma_{c u t}\right)$ depends upon the displacement column $q$ and its time derivatives as well as on the history of the surface being machined. It may be solved using Newmark's incremental scheme and a Newton-Raphson iterative method.

\subsection{Definition of Material Removal}

As we want and need to get an accurate description of regenerative forces due to previous motion of rake faces, we need a good modelling of the machined surface at each time step. This is necessary to insure a good coupling with the workpiece deformation.

To follow the geometry of the workpiece and get the chip section we introduce two geometry models, one for the workpiece and another one for the rake faces of the tool.

- For the workpiece, only the volume subjected to machining has to be modeled. We can use two types of representation: a B-Rep model based on a set of plane facets, or a volume dexel model (multi-level Z-Map models [34]) (Figure 2). The first one allows very fine description of machined surface, but leads to many difficulties to achieve a sufficient reliability, in particular when the tool is passing many time at the same place (this is often the case in milling). The second one, strongly reliable, has 


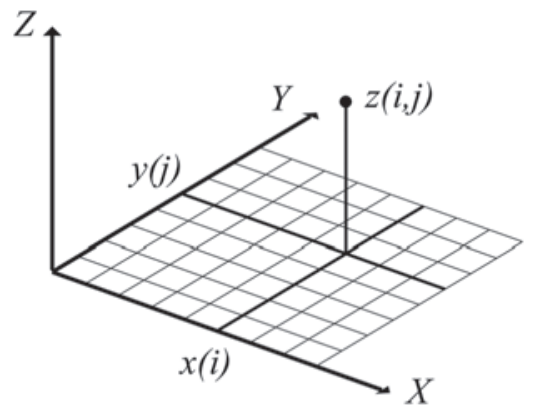

Z-Map model

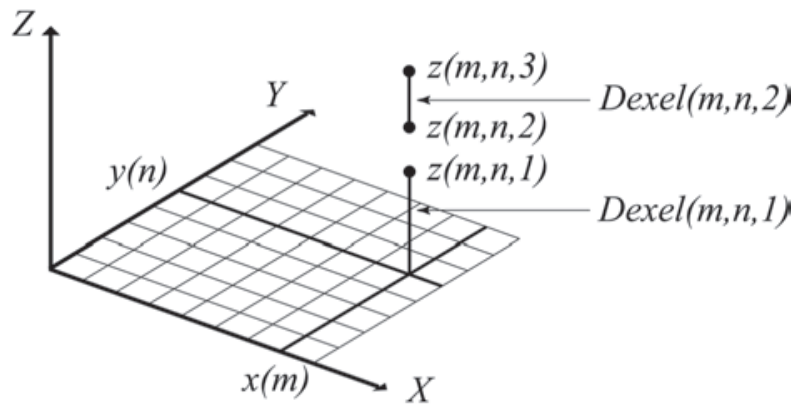

Multi-level Z-Map model

Figure 2. Z-map models

a precision which depends on the grid coarseness in the two directions normal to the Z-Map direction.

- For the surface of the rake faces we use a set of plane triangular facets.

In the following sections we focus on the dexel model for the workpiece.

\subsubsection{Definition of the machined domain}

Due to the simultaneous movements of the tool and the workpiece it is not straightforward to define the material removal. To achieve this goal it is necessary to choose a configuration where the workpiece does not move, i.e. a material configuration for the workpiece. In the dexel description, the material configuration is the reference configuration where the dexels remain straight and undeformed. This reference configuration is the only configuration where intersection algorithms can be defined easily with the dexel description.

To follow the deformation of the machined part of the workpiece we then need to define, at each time $t$, a one-to-one mapping ${ }^{t} \Phi_{W}$ from the reference (or material) configuration to the current configuration of the workpiece (2)(Figure 3 )

$$
\forall t, \quad \forall r M \in{ }^{r} \Omega_{W}(t), \quad \exists !{ }^{t} M \in{ }^{t} \Omega_{W}, \quad{ }^{t} M={ }^{t} \Phi_{W}\left({ }^{r} M\right)
$$

${ }^{t} \Omega_{W}$ is the current domain filled by the workpiece in the current (deformed) configuration at time $t$ and ${ }^{r} \Omega_{W}(t)$ is the reference domain filled by the workpiece in the material configuration at the same time. If a dexel description is not choosen, many equivalent choices are possible for the reference configuration, for example the initial configuration. The only restriction is the ability of doing the one-to-one mapping from the chosen reference configuration and the current configuration.

The position of each rake faces of the tool is defined in a same way by the one-to-one mapping ${ }^{t} \Phi_{T}(3)$ (Figure 3)

$$
\forall t, \forall{ }^{r} M \in{ }^{r} \Gamma_{T}, \quad \exists ! !^{t} M \in{ }^{t} \Gamma_{T}, \quad{ }^{t} M={ }^{t} \Phi_{T}\left({ }^{r} M\right)
$$

${ }^{t} \Gamma_{T}$ is the current surface filled by the rake faces at time $t$ and ${ }^{r} \Gamma_{T}$ is the reference surface describing the set of rake faces, for example the initial configuration of the rakes faces. ${ }^{r} \Gamma_{T}$ is time independent as we do not take into account wear in the present model.

We can then introduce ${ }^{r} V_{\text {generated }}$, the $3 \mathrm{D}$ domain generated by the displacement of the rake faces of the tool in material configuration. For a time interval $\left.] t_{1}, t_{2}\right]$, the material is then defined by the intersection: ${ }^{r} \Omega_{W}\left(t_{1}\right) \cap{ }^{r} V_{\text {generated }}\left(t_{1}, t_{2}\right)$. 

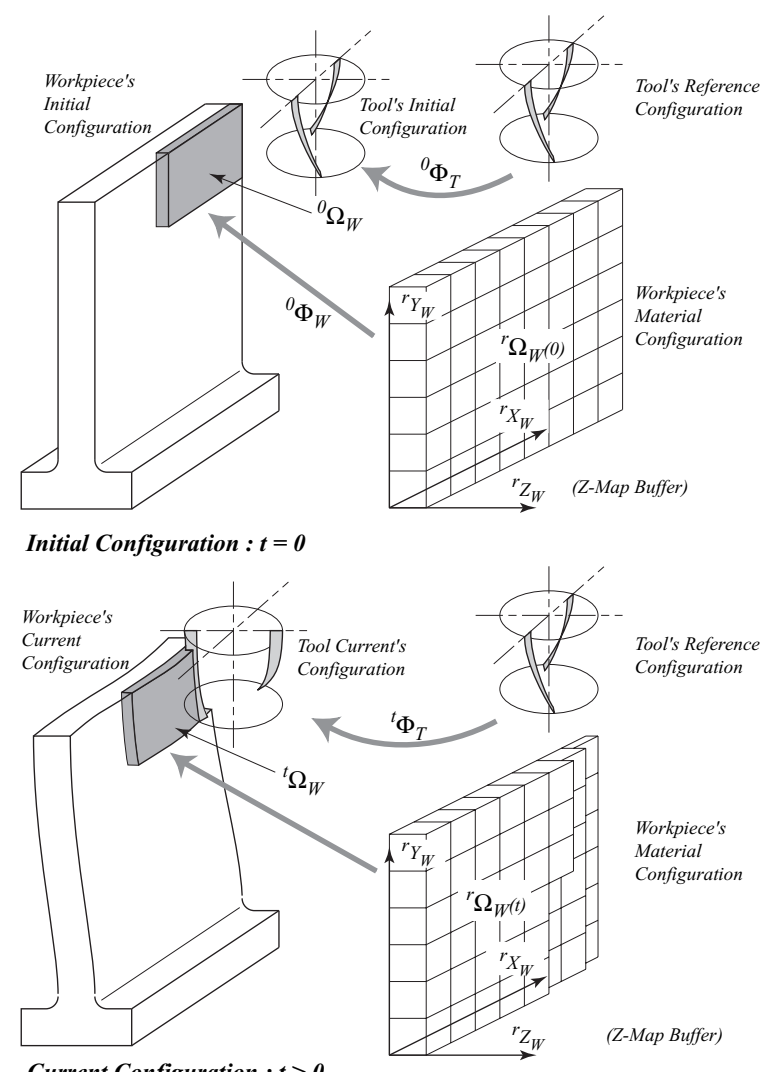

Figure 3. Piece and tool configurations

To carry the image of the rake faces in the material configuration we have to use $\left({ }^{t} \Phi_{W}\right)^{-1}$. Only a part $\Gamma_{c u t}$ of rake faces of the tool is included in the space filled by the workpiece $\Omega_{W}$. Thus, to transfer triangular facets of the geometric description of the rake faces which have only a potion inside the workpiece, an extension ${ }^{t} \Phi_{W}^{+}$of ${ }^{t} \Phi_{W}$ must be built.

${ }^{t} \Phi_{W}^{+}$is a one-to-one mapping verifying this only necessary condition:

$$
\forall t, \forall{ }^{r} M \in{ }^{r} \Omega_{W}(t), \quad{ }^{t} \Phi_{W}^{+}\left({ }^{r} M\right)={ }^{t} \Phi_{W}\left({ }^{r} M\right)
$$

This mapping is not unique and can be chosen conveniently in order to get an efficient implementation.

Once the extended mapping ${ }^{t} \Phi_{W}^{+}$is defined, the point of the rake faces of the tool can be calculated in the extended reference domain ${ }^{r} \Omega_{\text {extended }}$ for any time $t$. By construction ${ }^{r} \Omega_{W}(t)$ is included in ${ }^{r} \Omega_{\text {extended }}$.

$$
{ }^{r} V_{\text {machined }}\left(t_{1}, t_{2}\right)={ }^{r} \Omega_{W}\left(t_{1}\right) \cap{ }^{r} V_{\text {generated }}\left(t_{1}, t_{2}\right)
$$

and

$$
{ }^{r} V_{\text {generated }}\left(t_{1}, t_{2}\right)=\underset{\left.t \in] t_{1}, t_{2}\right]}{\operatorname{Sweep}}\left[\left({ }^{t} \Phi_{W}^{+}\right)^{-1}\left(\Gamma_{\text {cut }}(t)\right)\right]
$$




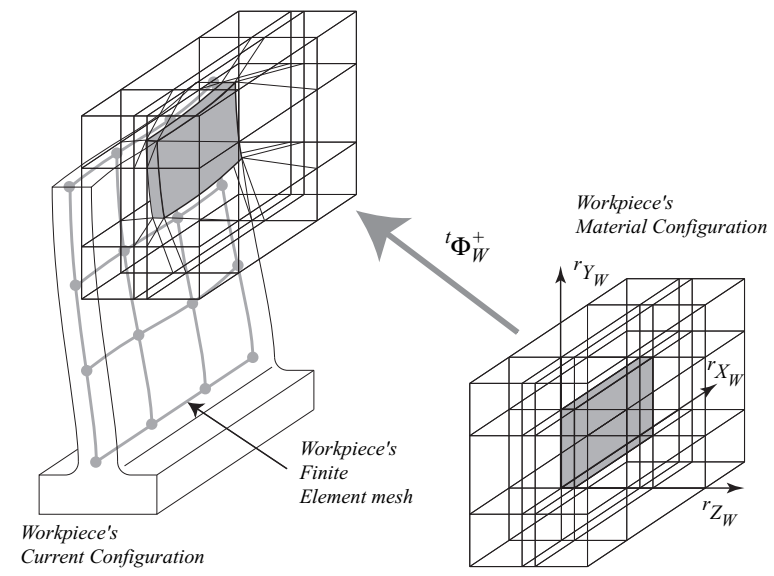

Figure 4. ${ }^{t} \Phi_{W}^{+}$construction - Hexahedron mesh

\subsubsection{Definition of $\Phi_{W}^{+}$}

Defining ${ }^{t} \Phi_{W}^{+}$by use of a Finite Element approach gives a systematic and simple process. As soon as thin-walled pieces are machined, the use of shell, or plate, finite element comes naturally. But, to maintain generality we introduce an hexahedron mesh, fully connected with the shell mesh, to define ${ }^{t} \Phi_{W}^{+} \cdot{ }^{t} \Phi_{W}^{+}$is constructed as follows:

- Construction of a regular hexahedron mesh in the material configuration in which the domain represented by the dexels is included. For example, if the domain represented by the dexel model is not too wide an unique hexahedron can be associated to it, an a set of 26 other hexahedrons surrounds it (Figure 4).

- The positions of the hexahedron's nodes, internal to the hexahedron mesh, are identified with respect of the shell, or plate, mesh of the piece in the undeformed configuration of the piece (initial configuration). This allows to construct the kinematic link

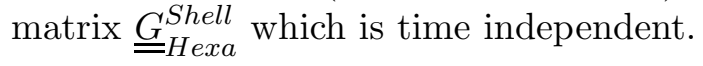

This matrix connects the column $\underline{q}_{H e x a}$, containing the kinematic parameters of the internal hexahedron's nodes ( 3 by nodes), to $\underline{q}_{\text {shell }}$, which contains the degrees of freedom of the shell mesh (7). $\underline{q}_{\text {shell }}$ is time dependent.

- The external nodes of the hexahedron mesh are fixed. They do not follow any motion.

- The knowledge of $\underline{q}_{\text {Hexa }}$ and a classical interpolation on the hexahedron mesh completes the definition of ${ }^{t} \Phi_{W}^{+}$

$$
\underline{q}_{\text {Hexa }}(t)=\underline{\underline{G}}_{\text {Hexa }}^{\text {Shell }} \cdot \underline{q}_{\text {shell }}(t)
$$

\subsubsection{Obtaining the workpiece final surface}

To achieve the simulation of the material removal we have to send back the points of the triangular facets of the rake faces in the material configuration with $\left({ }^{t} \Phi_{W}^{+}\right)^{-1}(6)$. At each iteration of the incremental scheme only the current coordinates of these points are known. Let be ${ }^{t} M$ one of theses points. The problem is then to find ${ }^{r} M$ such that:

$$
{ }^{t} M={ }^{t} \Phi_{W}^{+}\left({ }^{r} M\right)
$$


To solve this problem we have to find in which hexahedron is ${ }^{r} M$ and then a Newton iterative procedure is employed to find ${ }^{r} M$. Only a few number of iteration are required $(2$ or 3) because the deformations of the piece are very small and then ${ }^{r} M$ and ${ }^{t} M$ are close.

When this problem is solved for every point for the new position of the rakes faces it is possible to define ${ }^{r} V_{\text {generated }}\left(t_{1}, t_{2}\right)$ by its boundaries: the material position of the rake faces at $t=t_{1}$, the material position of the rake faces at $t=t_{2}$, and a triangular mesh that joint theses two sets of rake faces images (volume swept by the rake faces).

The intersection (boolean operation) between the workpiece domain and the domain generated by the rake faces enables then to follow the evolution of the workpiece geometry.

\subsection{Workpiece-Machine-Tool System Updates}

During machining, material removing induces a modification of rigidity and mass of the workpiece. This modification can generate a significant evolution of the dynamic behaviour of the piece if the latter is thin-walled. It is in particular the case for the thin shells where $2 / 3$ of the thickness can be removed during a roughing cut. The taking into account of the evolution of the behaviour is then necessary and must often be associated to the taking into account of the deformations of the workpiece in the machined zone (section 3 ).

A first possibility to take into account, as accurately as possible, the evolution of the machined domain is to adapt the finite element mesh after each step of time. That is unfortunately not possible for several reasons. It would be firstly necessary to have a sufficiently reliable meshing tool to be able to carry out simulation without spurious shutdowns, and sufficiently effective not to increase the calculating time in an exaggerated way. Then, a projection of the kinematic quantities (displacement and velocity) should be realized between the successive mesh what would generate a loss of information and additional costs. Finally, the constant evolution of the column of degrees of freedom $\underline{q}$ (evolution of the number of lines) would render the definition of $\Phi_{W}^{+}$more delicate to realize.

To overcome these difficulties, we propose to take into account in a simplified way the effect of material removal. The principal idea of this approach is to fix the geometry of the finite element mesh while modifying the mechanical characteristics (Young modulus $E$, density $\rho$ ) of the elements concerned with the removal thanks to a function of material presence $D(t, \underline{x}) ; \underline{x}$ is the position of a point belonging to one of the elements in the machined zone. As the vibrations remain small, with respect to the cut thickness, it is reasonable to assume that, at each time $t$, real removed volume is close to the theoretical removed volume (volume removed without any vibration). More precisely, it is possible to build, a each time $t$, a column $p(t)$ of values defining the theoretical position of workpiece and tool (without vibrations). The theoretical geometry can then be deduced, at a time $t$, from the history of $p(\tau)$ for $\tau \in[0, t]$. Under this circumstances $D(t, \underline{x})$ can be defined explicitly over all the simulation.

The correction on $E$ and $\rho$ is then defined by:

$$
E^{\prime}=E(1-D) \quad \rho^{\prime}=\rho(1-D) \quad \text { with } \quad 0<D<D_{\max }
$$

$D_{\max }$ is chosen equal to 0.95 to avoid singular matrices. The Poisson's ratio $\nu$ remains unchanged. The choice of a similar correction for the Young modulus and the density was retained not to generate spurious eigenvectors.

In section 3.3.1 we show, on a example, how $D$ rules the dynamic behaviour with comparison to a reference solution. In addition, due to the use of $D$, some values of the matrices $\underline{\underline{M}}, \underline{\underline{C}}, \underline{\underline{K}}$, are in constant evolution. In order to limit numerical costs, we present in section $3 . \overline{3.2}$ an efficient approximation which limits the number of factorizations of matrices within the framework of Newmark algorithm. 


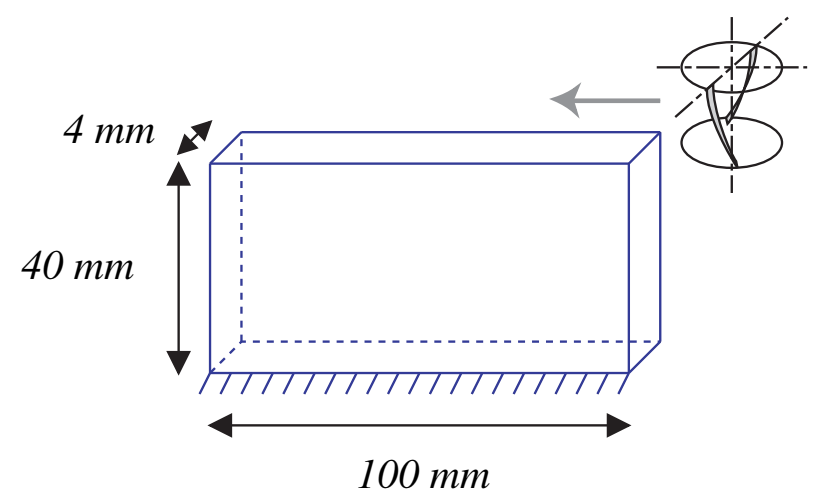

Figure 5. The workpiece before machining
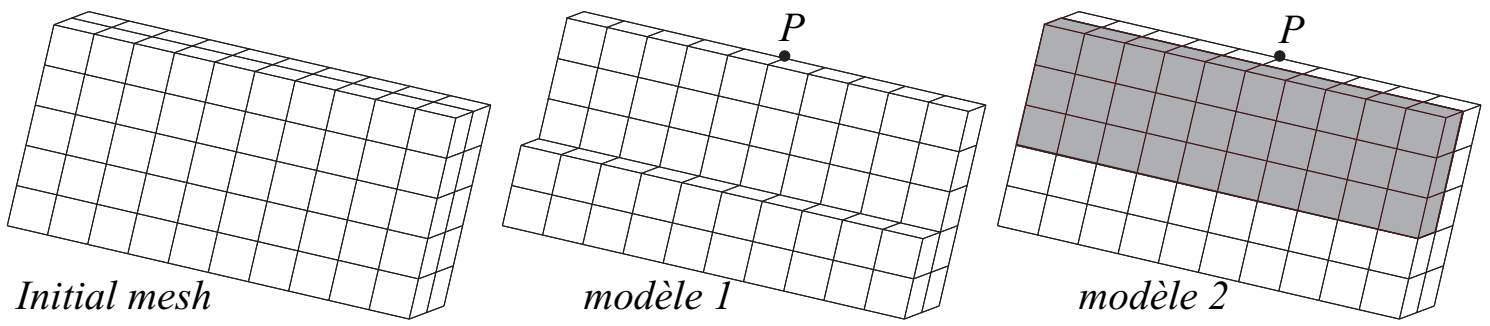

Figure 6. Meshes of the workpiece

\subsubsection{Validation of the use of $D$}

We compare here, for a finished machining, the dynamic behaviour of a model in which the "machined" elements are suppressed (model 1) with a model where these same elements are kept and managed via the function of material presence $D$ (model 2).

The machined workpiece (Figure 5) is a right-angled parallelepiped. Its dimensions are $100 \mathrm{~mm} \times 40 \mathrm{~mm} \times 4 \mathrm{~mm}$. The base of the piece is fixed. The material is a steel whose characteristics are as follows:

$$
E=2.10^{11} \mathrm{MPa} \quad \nu=0.3 \quad \rho=7800 \mathrm{kgm}^{-3}
$$

The mesh of the workpiece before machining contains 100 elements $(10 \times 5 \times 2)$. For the model 1, 30 elements are removed over the entire length $(10 \times 3 \times 1)$. For the model 2 , these same elements have a material presence function set to $D=0.9$. Machined volume relates to half the thickness, the $3 / 5$ the height and the whole length of the piece (Figure 6 ).

In Table 1, the first five natural frequencies for the two models are given.

\begin{tabular}{|c|c|c|c|}
\hline N of eigenvalue & Model 1 $(\mathrm{Hz})$ & Model 2 $(\mathrm{Hz})$ & Relative variation (\%) \\
\hline 1 & 2580 & 2529,6 & 1,95 \\
2 & 3221,6 & 3205,9 & 0,49 \\
3 & 5063,3 & 5119,8 & 1,11 \\
4 & 8272,1 & 8497,8 & 2,73 \\
5 & 13087 & 13723 & 4,86 \\
\hline
\end{tabular}

Table 1. Natural frequencies for the five first eigenvectors 
The natural frequencies for the two models are very close (relative variation is lower than $5 \%$ ) and so are shapes of the eigenvectors (no represented here). No spurious low frequency eigenvector is created in Modele 2 so the presence of material function seems to be an accurate approach.

\subsubsection{Limitation of the numerical costs}

We have chosen the Newmark time integration scheme to solve the equilibrium equation (1). Other integration scheme may be used without particular restrictions. Newmark's scheme $([35,36])$ can rapidly be described in the case of a constant time step $\Delta t$. The value of $\underline{q}$ at the time $t+\Delta t$ is then given by:

$$
\underline{\underline{K}}^{t+\Delta t} \underline{q}={ }^{t+\Delta t} \underline{Q}+{ }^{t} \underline{\bar{Q}}
$$

with

$$
\underline{\underline{\hat{K}}}=\frac{1}{\alpha \Delta t^{2}} \underline{\underline{M}}+\frac{\delta}{\alpha \Delta t} \underline{\underline{C}}+\underline{\underline{K}}
$$

and

$$
{ }^{t} \underline{\bar{Q}}=\underline{\underline{M}}\left\{a_{3}{ }^{t} \underline{\ddot{q}}+a_{2}{ }^{t} \underline{\dot{q}}+a_{0}{ }^{t} \underline{q}\right\}+\underline{\underline{C}}\left\{a_{5}{ }^{t} \underline{\ddot{q}}+a_{4}{ }^{t} \underline{\dot{q}}+a_{1}{ }^{t} \underline{q}\right\}
$$

and at last

$$
\begin{array}{lll}
a_{0}=\frac{1}{\alpha \Delta t^{2}} & a_{1}=\frac{\delta}{\alpha \Delta t} & a_{2}=\frac{1}{\alpha \Delta t} \\
a_{3}=\frac{1}{2 \alpha}-1 & a_{4}=\frac{\delta}{\alpha}-1 & a_{5}=\left(\frac{\delta}{2 \alpha}-1\right) \Delta t
\end{array}
$$

In the previous expressions, $\alpha$ and $\delta$ are parameters of the Newmark's scheme. For $\alpha=\frac{1}{4}$ and $\delta=\frac{1}{2}$ the method is unconditionally stable in a linear framework.

When there is material removal the matrix $\underline{\underline{\underline{K}}}$ changes because it depends on the machining evolution: $\underline{\underline{\hat{K}}}=\underline{\underline{\hat{K}}}(\underline{p}(t))$. In the case described in the preceding paragraph, the advance of the tool, a milling-cutter, is described thanks to a single scalar: $\underline{p}(t)=p(t)$.

In setting

$$
\underline{q}={ }^{t+\Delta t} \underline{q} \quad ; \quad p=p(t+\Delta t) \quad ; \quad \underline{Q}_{t o t a l}={ }^{t+\Delta t} \underline{Q} \quad+\quad{ }^{t} \underline{\bar{Q}}
$$

the expression (11) can be rewritten in the following form:

$$
\underline{\underline{\hat{K}}}(p) \quad \underline{q}=\underline{Q}_{t o t a l} \quad \text { or } \quad \underline{q}=\underline{\underline{\hat{K}}}^{-1}(p) \quad \underline{Q}_{\text {total }}
$$

The factorization of $\underline{\underline{\hat{K}}}\left(\underline{p}(t)\right.$ ) (a Crout factorization $\underline{\underline{L}} \cdot \underline{\underline{D}} \cdot \underline{\underline{L}}^{t}$ ) to each time step represents an important numerical cost. To limit this numerical cost it is possible to approximate, on an interval of time $\left[t_{i-1}, t_{i}\right]$, the inverse of the matrix $\underline{\underline{\hat{K}}}$ by the linear combination of two matrices evaluated at the times $t_{i-1}$ and $t_{i}$ :

$$
\forall t \in\left[t_{i-1}, t_{i}\right] \quad \underline{\underline{\hat{K}}}^{-1}(p(t))_{\text {approx }}=(1-\eta) \underline{\underline{\hat{K}}}^{-1}\left(p_{i-1}\right)+\eta \underline{\underline{\hat{K}}}^{-1}\left(p_{i}\right)
$$

where $p_{i-1}=p\left(t_{i-1}\right), p_{i}=p\left(t_{i}\right)$, and $\eta=\eta(t)=\frac{t-t_{i-1}}{t_{i}-t_{i-1}}$.

In setting

$$
\underline{q}_{i-1}=\underline{\underline{\hat{K}}}^{-1}\left(p_{i-1}\right) \underline{Q}_{t o t a l} \quad \underline{q}_{i}=\underline{\underline{\hat{K}}}^{-1}\left(p_{i}\right) \underline{Q}_{\text {total }}
$$


$\underline{q}_{a p p r o x}$ is obtain in an obvious way:

$$
\underline{q}_{\text {approx }}=(1-\eta) \underline{q}_{i-1}+\eta \underline{q}_{i}
$$

With this approach, at each increment, only two linear resolutions with the factorised form of $\underline{\underline{\hat{K}}}$ are to be carried out $\left(\underline{q}_{i-1}\right.$ and $\underline{q}_{i}$ calculation). With each new interval of time $\left[t_{j-1}, t_{j}\right]$ the factorization of $\underline{\underline{\hat{K}}}\left(p_{j}\right)$ must also be done. The number of time steps per interval $\left[t_{j-1}, t_{j}\right]$ is chosen as large as possible, taking into account the more or less large variations of the mechanical characteristics due to the theoretical material removal.

In order to validate the approach, that we will call simplified approach, we present two different simulations working on the support given on the Figure 5.

- Simulation 1: the FE matrices are recomputed after each time step $\Delta t$ according to parameter $p(t)$.

- Simulation 2: the FE matrices are only recomputed at selected time steps. The interpolated inverse of $\underline{\underline{\hat{K}}}$, on the intervals, is thus introduced in this case. It uses the simplified approach.

To simplify the study, we took a simple sinusoidal force of form $F(t)=\cos (\omega t)$ to make the validation. This force, which appears in the vector $q(t)$, follows the advance of machining and is applied to the higher part of the workpiece. The parameters of simulation are such as traversing the piece over all its length takes 10000 increments. The time step $\Delta t$ equal to $10^{-4} s$.

\begin{tabular}{|c|c|c|}
\hline Number of intervals & Number of increments per interval & $E(t)$ Max \\
\hline 100 & 100 & $1,975.10^{-2}$ \\
50 & 200 & $1,077.10^{-1}$ \\
20 & 500 & $4,945.10^{-1}$ \\
10 & 1000 & 1,660 \\
5 & 2000 & 5,260 \\
1 & 10000 & 63,111 \\
\hline
\end{tabular}

Table 2. Relative error between simplified and reference approaches (in percent)

To compare the results of the simplified approach with respect to the results of the reference solution, we calculated a relative error $E(t)$ (equation (21)) in a point $P$ of the piece belonging to the mesh (Figure 6). As it is visible on the figure, the point $P$ is selected in the not machined part of the workpiece. The evolution of displacements at $P$ point (for the reference solution) is given Figure 7. Table 2 gathers the maximum errors relative $E(t)$ at point $P$. From 100 increments by interval, we obtain small relative error $(2 \%)$ what shows the interest of the approach

$$
E(t)=\frac{\left|q_{\text {Ref }}-q(t)\right|}{\left|q_{\text {RefMax }}\right|}
$$

Parameters of the simulations:

- The frequency of the first eigenvector of the unmachined workpiece is $f=3342,68 \mathrm{~Hz}$. We took for $\omega$ the value of the first natural angular frequency: $\omega=21002,66 \mathrm{rad} / \mathrm{s}$ ). 


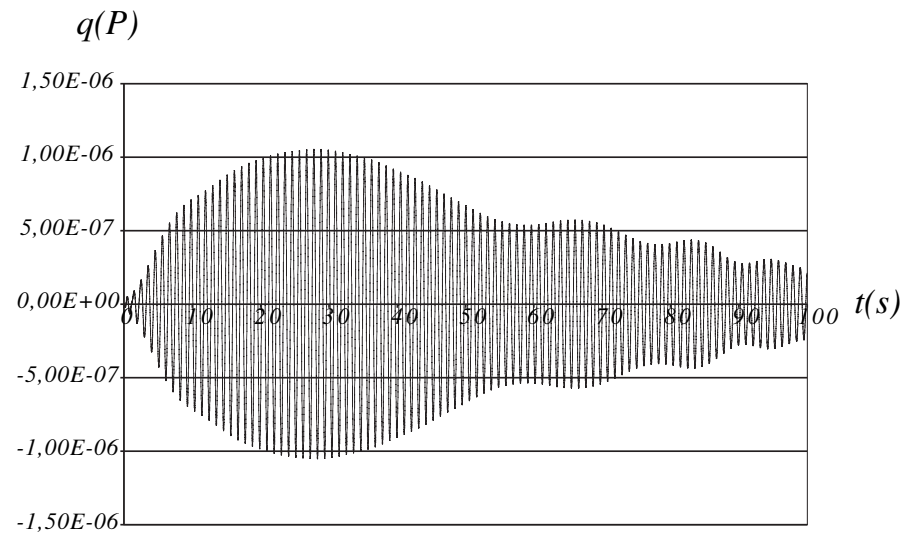

Figure 7. Displacement evolution at point $P$

- The damping matrix $\underline{\underline{C}}$ is chosen to be proportional to the stiffness matrix $\underline{\underline{K}}: \underline{\underline{C}}=$ $\lambda \underline{\underline{K}} . \lambda$ was computed such that a damping ratio of 0,01 is obtained for first eigenvector of the not machined workpiece. This leads to $\lambda=2(0,01 / \omega) \sim 9.10^{-7} s$.

- The longitudinal speed of the tool is taken to be $1 \mathrm{~m} / \mathrm{s}$. This is not very representative of a speed under real conditions which is a little below $0.1 \mathrm{~m} / \mathrm{s}$. This choice was made to limit the calculating times.

\subsection{Overview of the Algorithm at the Macroscopic Scale}

A recapitulation of the principal steps for a whole simulation is given in Box 1. As shown in this diagram, the proposed approach needs two incremental loops and two iterative loops.

\subsection{Example of Machining of thin Walled Pieces}

To conclude this chapter we show an example of the machining of a thin walled workpiece. The geometry of the workpiece is that described on Figure 5. As shown on Figure 8, the machined domain has $18 \mathrm{~mm}$ of axial depth of cut (along the $y$ axis), and the radial depth of cut is fixed to $1.0 \mathrm{~mm}$ (in the $z$ direction). The cutter is a helical end mill with 3 teeth, a constant pitch angle of 30 degrees, and a diameter of $12 \mathrm{~mm}$. Feed per tooth is $1.8 \mathrm{~mm}$ per revolution which give $0.1 \mathrm{~mm}$ of roughness.

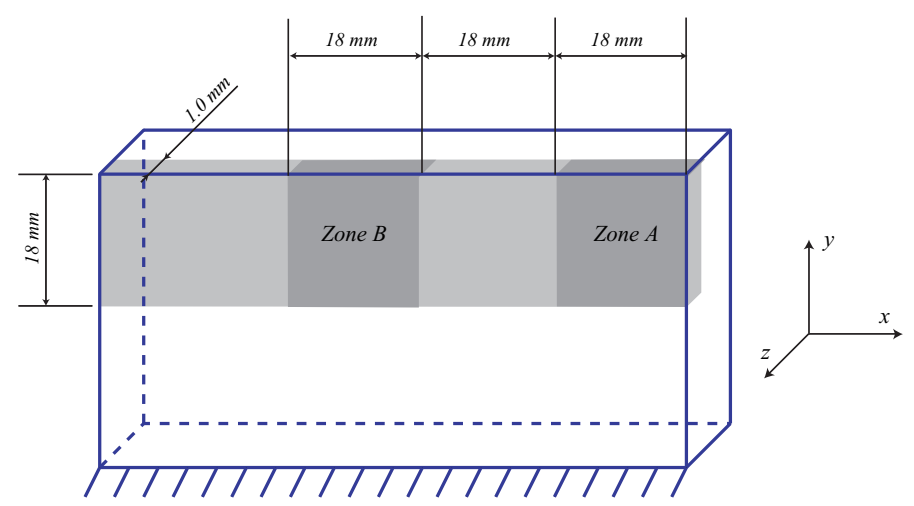

Figure 8. Definition of the machined domains for a thin-walled worpiece 
(i) Initialisation

- Meshing of the workpiece (for example with plate or shell elements)

- Identification of region subjected to machining

- Choice of the Dexels parameters: number, orientation

- Creation of the hexahedron mesh and construction of $\underline{\underline{G}}_{\text {Hexa }}^{\text {Shell }}$ for ${ }^{t} \Phi_{W}^{+}$

- Computation and factorisation of $\underline{\underline{\hat{K}}}\left(p_{0}\right)$, computation of other initial matrices

(ii) Loop over all time intervals

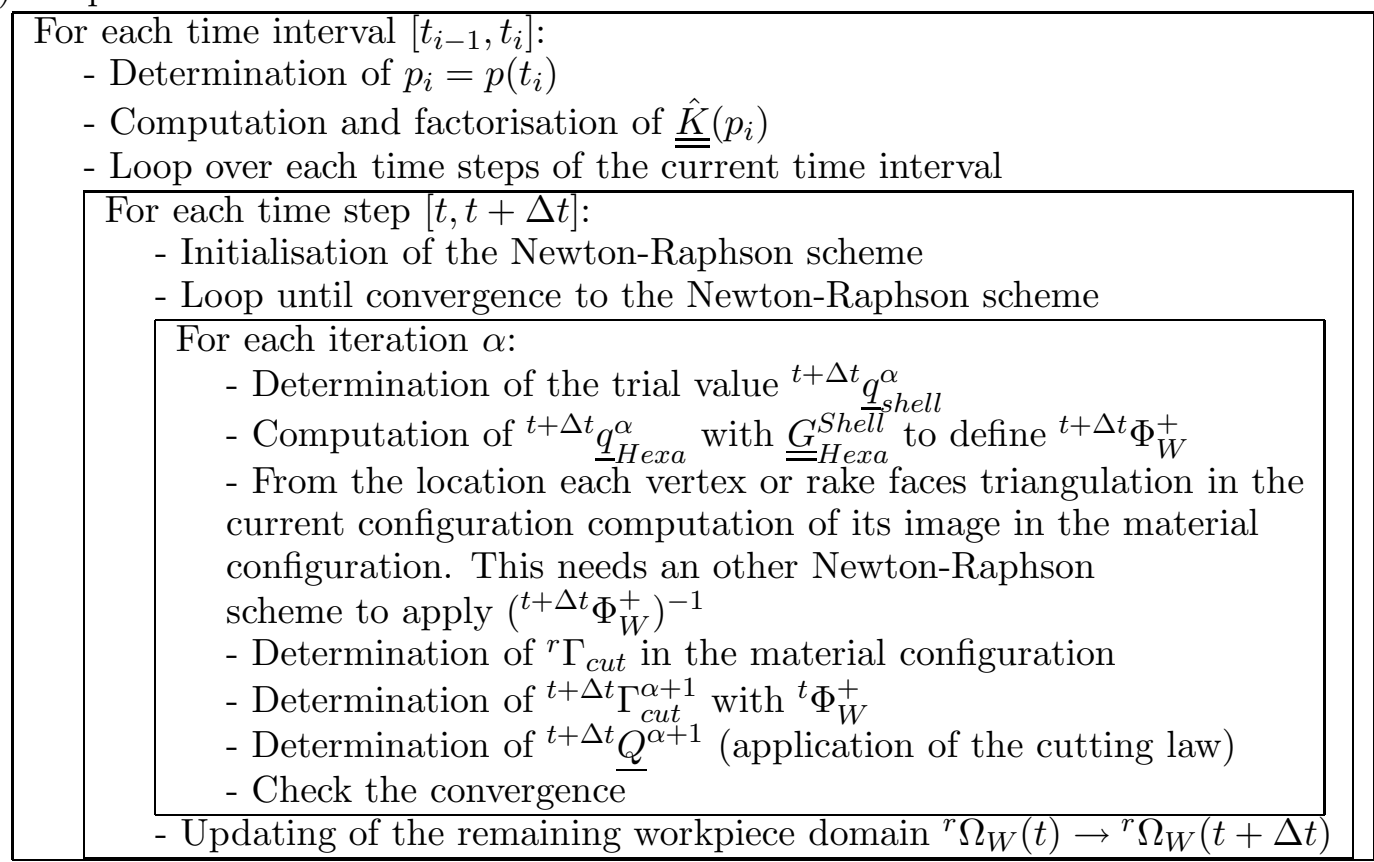

(iii) Outputs and post processing

Box 1. Principal steps of the algorithm at the macroscopic scale

The workpiece is modelled with a single 8 nodes plane shell element. The first natural frequency of this plate is $2134 \mathrm{~Hz}$. Spindle angular velocity leads to a frequency of excitation $(2155 \mathrm{~Hz})$ very close to the first natural frequency of the workpiece. The damping matrix $\underline{\underline{C}}$ is chosen to be proportional to the stiffness matrix $\underline{\underline{K}}: \underline{\underline{C}}=\lambda \underline{\underline{K}} . \lambda$ was computed such that a damping ratio of 0,005 is obtained for first eigenvector. This leads to $\lambda=2(0,005 / \omega) \sim$ $7.510^{-7} \mathrm{~s}$.

A dexel description is used for the machined domain. We have put 1400 dexels along the $x$ axis and 180 along the $y$ axis.

Figure 9 shows, on the left, the machined surface for a rigid workpiece. The two other pictures correspond to the machined boundaries for zone A and B (defined on Figure 8) for the flexible workpiece (Young modulus $E=2.110^{11} \mathrm{~Pa}$, density $\rho=7800 \mathrm{~kg} / \mathrm{M}^{3}$, Poisson's ratio $\nu=0.3)$.

For zone $A$ the roughness is equal to $0.88 \mathrm{~mm}$, whereas for zone $B$ the roughness is equal to $0.30 \mathrm{~mm}$, both having to be compare with the roughness of the rigid workpiece: $0.10 \mathrm{~mm}$. It is clear that, for this example, the flexibility of the workpiece plays a very important 


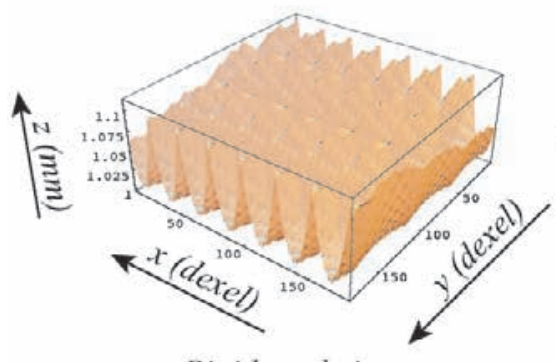

Rigid workpiece

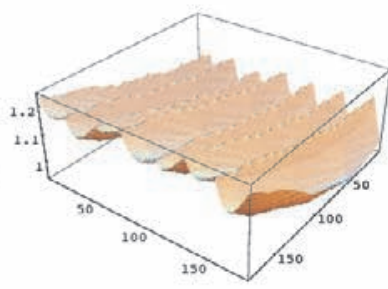

Fexible workpiece Zone B

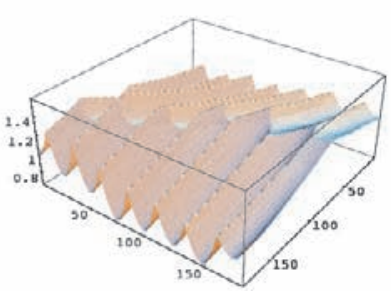

Flexible workpiece Zone A

Figure 9. Example of surface quality simulation for a thin-walled worpiece

part. The important variation of the roughness between zone $A$ and zone $B$ shows the importance of the taking into account of the deformation of the workpiece.

\section{NUMERICAL SIMULATION AT THE MESOSCOPIC SCALE}

\subsection{Goals and Context of Simulations at the Mesoscopic Scale}

The main goals of numerical simulations at the mesoscopic scale are to determine:

- the thermomecanical solicitations applied on the tool in order to predict and/or take into account tool wear and failure/fracture (which both are strongly dependent on temperature),

- the residual stress and chemical composition inside the machined surface layer; this results of phase transformations and applied strain and strain rate during machining,

- the shear-band formation, and strain localization in order to predict the chip flow and morphology: continuous, segmental, or serrated chip formation can be found,

- macroscopic cutting forces evolution in the case of continuous (turning) or discontinuous cutting (milling or blanking).

All theses points are dependent upon the cutting conditions: workpiece parameters (material type, crystallography, temperature, pre-deformation), cutting tool parameters (tool design geometry, material, coated or uncoated carbides), and cutting parameter (speed, feed, depth of cut, lubrification) (Figure 10). A typical study of the strains, strain rates and temperature during chip formation is, for example, performed by Jazspers and Dautzentberg in [37].

The simulation at the mesoscopic scale allows parametric studies of the cutting processes in order to optimize cutting conditions. It allows to check the state of the machined or tool material everywhere and at any instant of the cutting process. It is the only way to improve the understanding of the material cutting mechanisms, especially at in high speed machining where experimental data are extremely difficult to obtain.

To achieve theses goals the mechanical frame for the simulations is the following:

- a model of the machined part of the workpiece as a homogeneous continuum media, or a piecewise continuum (if inclusions have to be taken into account); however, for micro-machining, some recent approaches use discrete models such as molecular dynamics $[38,39]$ and are today limited by the number of cells to avoid a too important computational time, 


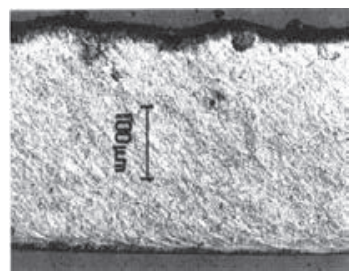

Continuous Cutting speed : $100 \mathrm{~m} / \mathrm{s}$

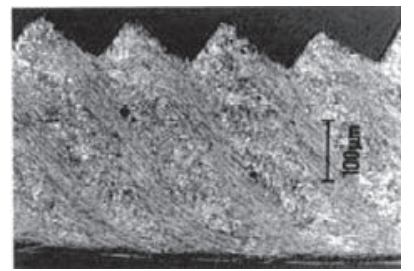

Serrated

Cutting speed : $300 \mathrm{~m} / \mathrm{s}$

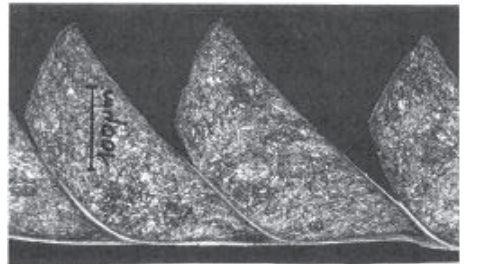

Quasi-discontinuous

Cutting speed : $1000 \mathrm{~m} / \mathrm{s}$

Figure 10. Example of chip morphology dependence on the cutting speed

- large transformations with high strain and high strain rates,

- different kind of heat-generation: by friction between the tool and the workpiece, by plastic deformations inside the workpiece material (in particular in the chip),

- different kind of heat-transfer: heat conduction across contact zones, radiation, free convection along external surfaces for the workpiece and the tool,

- thermomechanical coupling; in particular thermal softening,

- and if needed, phase transformations and chemical diffusions.

Two kinds of constitutive models have to be introduced:

- surface models for friction, heat repartition between workpiece and tool, wear, ...

- volume models for material behaviour which can be thermoelasto-plastic, thermoviscoplastic, elastic-plastic, viscoplastic, rigid-plastic, with or without damage, ...

The choice and/or the construction and/or the identification of the constitutive law is of a particular difficulty:

- it must be as close as possible to material behaviour on a wide range of parameter variation: up to 5 for the strain, up to $10^{4} s^{-1}$ for the strain rates and for temperature from the room temperature to the melting point (from about $293 K$ to $1000 K$ for standard material and conditions),

- the observation of the physic phenomenon during the cutting process must be done on volume of only a few $\mathrm{mm}^{3}$, whereas the relative speed between the workpiece is of the order of $10 \mathrm{~m} / \mathrm{s}$ for high speed machining or up to $30 \mathrm{~m} / \mathrm{s}$ for high speed blanking.

Once the constitutive law is selected and identified, the numerical implementation is not of major difficulty even if it is not easy because of the large transformations context.

As for all simulations, the quality, the quantities and the predictability of results are directly dependent upon the choice of the previous models and the resolution algorithms.

\subsection{Overviews of the Current Approaches}

The first approaches in the modeling of cutting processes where applied on machining in a analytical way by Ernst and Merchant [40, 41, 42] on orthogonal cutting. Recent publications still concern the analytical approaches and give more accurate results on cutting forces $[43,44,45]$ in different kind of cutting conditions [46]. Some of them focuse on specific aspects such as the white layer apparition [47] or the burr formation [48]. At least, 
hybrid analytical numerical approaches also exist; for example Marinov uses this type of solution to determine the shear angle [49].

Nevertheless, none of theses analytical solutions are able to give detailed 3D solutions for stresses, strain, temperature, or to predict the chip morphology. Their main interest is the construction of cutting laws in various cutting conditions for the simulations at the macroscopic scale.

Among the numerical procedures, the finite-element method (FEM) was the first $[50,51$, $52,53]$, and still remains the most frequently used. A review of the pioneer work in this field can be found in $[54,55]$. The FE approaches are bi-dimensional $(2 \mathrm{D})$ or a three-dimensional (3D) and differentiated by:

- the choice of the constitutives models,

- the nature of the FE approach: Eulerian, Lagrangian, or Arbitrary Lagrangian Eulerian (ALE),

- the adopted strategy to simulate de material separation: simply removing the elements ("killing elements") where the strains or strain rates are considered excessive, or using a separation line (surface in 3D simulations) as in fracture mechanism. The separation line may be given a priori or not, and if it is not the case an adaptive mesh strategy must be introduced in order to simulate the material separation flow near the tool tip.

The Johnson-Cook constitutive model is the more frequently used. This thermo-viscoplatic law (64) is base on a multiplicative form. It allows to take into account the main experimental phenomena [56]. In [57] a table of coefficients is given for this law for various materials. Authors made modifications to the Johnson-Cook model in order to introduce in a more accurate way the observed hardening, for certain materials, when the temperature increases $[58,59]$. Finally a modeling of the damage was also proposed by Johnson and Cook in a similar form [60].

Many other laws exist:

- the thermo-elastic-plastic law proposed by Z.-C. Lin and S. Y. Lin [61] is also used by many authors; Usui et Shirakashi [62], Zone-Chin Lin and Yeou-Yih Lin [63], Mamalis et all [64], Yen et all [65],

- the rigid thermo-viscoplasic law defined by J. S. Wu et all [66] is, for example, used in [67],

- the Lemonds Needleman law [68] was used by Marusich and Ortiz to make one of the first 2D simulations of orthogonal cutting with segmented chip [69],

- the law of Lei, Shi et Incropera [70] used par themselves [71], ...

Other constitutive laws can be found in the material database for manufacturing simulation is proposed by J. Söder and T. Altan [72] or in the PhD thesis of Firas Ali [73].

For the sake of simplicity, one of the first approach used for simulating chip formation was Eulerian [74]. Firstly, only continuous chip were simulated by such an approach but usually the chips are not continuous. Recently, an extended eulerian procedure, the multimaterial finite element formulation, was applied to high speed machining simulation with segmented chip [75]. The use of lagrangian approaches is indeed the most frequent way to simulate any type of chip morphology. One of the main difficulties for theses approaches is the treatment of material separation near the tool tip. Again, for the sake of simplicity a predefined split line was introduced. The first authors who used such approach where 
Strenkowski and Carroll [74, 76]; they used a plastic strain criterion to separate the nodes on this line. Many developments were carried out then in this direction with various criteria of elements separation $[77,78,79,80,81,63,64,67,82,65,83,84]$.

The main drawback of the technique including a line separation is their inefficiency in predicting the phenomenon near the tool tip, where the higher solicitations are indeed present. To follow the material separation, adaptive meshing method were introduced by Camacho et all [85] and used by Marusich and Ortiz in a 2D analysis of metal cutting with a fracture stress criterion used to define split lines [69]. An more simple splitting technique is based on a damage criterion coupled with the deletion of the elements when the limit is reached [86, 87, 88, 89]. At last, continuous remeshing without any damage or fracture criterion can be used near the tool tip to separate the material [65]. Today, 3D simulations are available with these kinds of technique with the Deform3D code [90] and example of simulations are given in [91].

The Arbitrary Lagrangian Eulerian formulation (ALE) offers an alternative to the full Lagrangian description. In this formulation the grid points are not constrained to remain fixed in space (as in the Eulerian description) or to move with material points (as in Lagrangian description), but have their own equations describing the motion. The ALE formulation was firstly applied on cutting simulation by Rokotomalala et all [92]. The ALE approach has also been used by Olovsson et al. [93] in a two-dimensional finite element model of orthogonal metal cutting. Guo et al. use it for 2D segmented chip formation with a kill element technique [87], and Pantalé et al. [88] still use it on 2D and 3D numerical models of metal cutting with damage effects (Johnson-Cook damage model is used [60]).

$3 \mathrm{D}$ simulations can be achieve with ALE approches but, if a reduction of the finite element mesh distortions is present, the definition of the motion between the grid/mesh and material points is not trivial.

\subsection{Typical Applications of Numerical Simulation on Machining}

Two typical applications of the cutting simulation are used for evaluating the technical choices (in order to improve the numerical strategies) and the practical use of simulation to study the process.

Concerning the first aspect, the simulations are focusing on the influence of simulation parameters such as the flow stress [94], the thermal conductivity [95], or influence of the hourglass treatment [89] on the chip morphology (curvature, thickness, segmentation).

In the second one, process parameters are tested. The accuracy and efficiency of the machining process modeling is sufficient to give interesting results about:

- the influence of the rake angle in orthogonal cutting [81],

- tool wear in turning operations [84],

- comparison of different tool edge geometries and their influence on chip formation [65],

- residual stress [83].

\subsection{Remaining Difficulties}

Today many commercial softwares are able and used to do simulations at the mesoscopic scale (MSC/Marc, Abaqus/Explicit [96] and Abaqus/Standard [97], Deform2D and Deform3D [90], AdvantEdge ${ }^{T M}$ [2], LS-Dyna [98]). Comparison of various codes can be found, as in [99] between i.e. MSC-Marc and AdventEdge in 2D. The main interest of the commercial codes is their ability to solve problems involving an important number of different features as it is the case for material cutting simulations (thermomechanical problem with 
large strain and strain rates under contact). However, difficulties still remain in the modeling of the physical phenomenon involved in the high speed cutting processes, and in the monitoring of the mesh evolution in 3D finite element analysis.

It should be noted that inverse approaches [100] are an appealing choice in order to find the coefficients of the constitutive laws used in machining as they use an important number of parameters. As inverse approaches are based on repetitive comparison between numerical simulation and experimental data, numerical simulation need to be accurate and efficient.

The evaluation of solutions to solve the encountered difficulties is however a difficult task in most of the commercial codes in which many parts are insufficiently open, even inaccessible. In many cases the use to research softwares, even if their potential is limited, is mandatory. In the next section we propose a original alternative, implemented in a research code, to simulate large deformations without the difficulties inherent to the finite element remeshing. The proposed "mesh-free" approach, based on a Voronoi diagram and associated Voronoi cells, uses specific data structures. For example, the number of degrees of freedom is variable for one Voronoi cell to another which makes the use of pre-existing code very delicate.

\section{A NEW APPROACH FOR LARGE DEFORMATION: THE C-NEM METHOD}

In this section, the interest of meshfree techniques in the context of mesoscopic simulations of machining is presented. The meshless simulations are illustrated in the context of large strains nonlinear elastoplastic, including dynamics, thermomechanical coupling, as well as localization in the chip formation. For this purpose, the constitutive equations of the model as well as algorithmic procedures is briefly described. The weak form of conservation laws is then discretized using the constrained natural element method (C-NEM). The technique is presented, and some of its attractive features for cutting simulation, i.e. the possibility to easily handle discontinuities or refinement, are first illustrated through benchmarks and then applied to the simulation of blanking and chip formation with shear localization. A first example of three-dimensional oblique cutting is presented. The purpose of these preliminary results is to show the potential of the technique, and need further improvements.

\subsection{Formulation of the Coupled Thermo-Mechanical Problem}

\subsubsection{Preliminaries}

In this section, we briefly summarize some fundamental kinematic relations and introduce the appropriate notations. We consider two configurations of a body $B$ : the first one, the reference configuration $\Omega_{0} \subset \Re^{d i m}$ with external boundary $\partial \Omega_{0}$ (not necessarily the initial configuration), where $\mathbf{X}$ denotes the coordinates of a point in this configuration. The second, called current configuration, is denoted by $\Omega_{x} \subset \Re^{d i m}$ with external boundary $\partial \Omega_{x}$ at time $t$, with $\mathbf{x}$ the coordinates of a point in the current configuration. $\mathbf{x}$ is related to $\mathbf{X}$ by:

$$
\mathbf{x}=\mathbf{X}+\mathbf{u}(\mathbf{X}, t)
$$

the deformation gradient is defined by:

$$
\mathbf{F}=\frac{\partial \mathbf{x}}{\partial \mathbf{X}}=\mathbf{1}+\nabla_{\mathbf{X}} \mathbf{u}, \quad J=\operatorname{det}(\mathbf{F})>0
$$

with $\operatorname{det}(\mathbf{F})$ being the determinant of $\mathbf{F}$. From now on, we use a hyperelastic formulation based on the multiplicative decomposition of the deformation gradient, with elastic response 
described by a hyperelastic stored energy function. In this context, elastic predictor becomes exact and the need for incrementally objective algorithms is entirely avoided.

The basic hypothesis underlying this approach to finite strain elastoplasticity is the multiplicative split of the deformation gradient, $\mathbf{F}$, into elastic and plastic parts:

$$
\mathbf{F}=\mathbf{F}^{e} \mathbf{F}^{p}
$$

This assumption, firstly proposed by Lee [101], admits the existence of a local unstressed intermediate configuration. Following the multiplicative split of $\mathbf{F}$, the velocity gradient, $\mathbf{L}=\dot{\mathbf{F F}}^{-1}$ can be decomposed additively as

$$
\mathbf{L}=\mathbf{L}^{e}+\mathbf{L}^{p}
$$

where $L^{e}$ and $L^{p}$ are, respectively, the elastic and plastic contributions defined by:

$$
\mathbf{L}^{e}=\dot{\mathbf{F}}^{e}\left[\mathbf{F}^{e}\right]^{-1}, \quad \mathbf{L}^{p}=\mathbf{F}^{e} \dot{\mathbf{F}}^{p}\left[\mathbf{F}^{p}\right]^{-1}\left[\mathbf{F}^{e}\right]^{-1}
$$

Similarly, the stretching tensor, $\mathbf{D}:=\operatorname{sym}[\mathbf{L}]$, can be decomposed as:

$$
\mathbf{D}=\mathbf{D}^{e}+\mathbf{D}^{p}
$$

where $\operatorname{sym}($.$) is the symetric part of (.), and the elastic and plastic stretching tensors given$ by:

$$
\mathbf{D}^{e}=\operatorname{sym}\left[\mathbf{L}^{e}\right], \quad \mathbf{D}^{p}=\operatorname{sym}\left[\mathbf{L}^{p}\right]
$$

\subsubsection{Hyperelastic constitutive law}

From polar decomposition, $\mathbf{F}^{e}$ is given by

$$
\mathbf{F}^{e}=\mathbf{R}^{e} \mathbf{U}^{e}
$$

where $\mathbf{U}^{e}$ and $\mathbf{R}^{e}$ are, respectively, the elastic right stretch tensor and the elastic rotation.

Let $\boldsymbol{\epsilon}^{e}$ denote the Eulerian (or spatial) logarithmic strain tensor [102]

$$
\boldsymbol{\epsilon}^{e}=\ln \left[\mathbf{U}^{e}\right]
$$

where $\ln [$.$] above denotes the tensor logarithm of (.) which involves spectral decomposition$ of $\mathbf{U}^{e}$. Following Peric et al. [103], we assume the existence of a quadratic strain energy function $\psi^{e}\left(\boldsymbol{\epsilon}^{e}\right)$ in the form of a scalar symmetric function of its stretches $\lambda_{i}(i=1,2,3)$ given by:

$$
\psi^{e}\left(\lambda_{1}^{e}, \lambda_{2}^{e}, \lambda_{3}^{e}\right)=\mu\left[\ln \left(\lambda_{1}^{e}\right)^{2}+\ln \left(\lambda_{2}^{e}\right)^{2}+\ln \left(\lambda_{3}^{e}\right)^{2}\right]+\frac{1}{2} \lambda\left(J^{e}\right)^{2}
$$

where $\mu$ and $\lambda$ are Lamé's parameters and $\left(J^{e}\right)=\lambda_{1}^{e} \lambda_{2}^{e} \lambda_{3}^{e}$ is the Jacobian. After applying standard procedure, the following hyperelastic constitutive equation is obtained:

$$
\mathbf{T}=\frac{\partial \psi^{e}}{\partial \boldsymbol{\epsilon}^{e}}=\mathbf{C}^{e}: \boldsymbol{\epsilon}^{e}
$$

where $\mathbf{T}$ is the rotated stress tensor. Assuming incompressibility of the plastic flow, it is expressed as:

$$
\mathbf{T}=\left[\mathbf{R}^{\mathrm{e}}\right]^{T} \boldsymbol{\tau} \mathbf{R}^{e}
$$

where $\boldsymbol{\tau}=J \boldsymbol{\sigma}$ is the Kirchhoff stress tensor, $\mathbf{C}^{e}$ is the fourth-order isotropic elastic tensor. Further details about the thermo mechanical foundations can be found in [104]. 


\subsubsection{Numerical integration of the constitutive equations}

Typically, within an incremental numerical procedure for solving history dependent problems, a numerical approximation to the material constitutive law is needed to update stresses $\boldsymbol{\tau}$ as well as the internal variables $\alpha \equiv \bar{\epsilon}^{p}$ (equivalent plastic strain) within each time (load) increment. In the present context, given the values of the variables $\left\{\boldsymbol{\tau}_{n}, \mathbf{F}_{n}^{p}, \alpha_{n}\right\}$ at the beginning of a generic increment $\left[t_{n}, t_{n+1}\right]$, an algorithm is required to update $\left\{\boldsymbol{\tau}_{n+1}, \mathbf{F}_{n+1}^{p}, \alpha_{n+1}\right\}$ at the end of the increment.

Under the assumption of elastic isotropy, the elastic Eulerian logarithmic strain tensor is updated according to:

$$
\boldsymbol{\epsilon}_{n+1}^{e}=\boldsymbol{\epsilon}_{n+1}^{e \text { trial }}-\Delta \gamma \mathbf{N}_{n+1}
$$

which has the same format that the standard return mapping used in the infinitesimal theory $[104,105,106,107]$.

$$
\mathbf{N}_{n+1}=\frac{3}{2} \frac{\operatorname{dev}\left(\mathbf{T}_{n+1}\right)}{J_{2}\left(\mathbf{T}_{n+1}\right)}
$$

where $J_{2}($.$) is the second invariant of (.). The trial elastic logarithmic strain \boldsymbol{\epsilon}_{n+1}^{e \text { trial }}$ is given by:

$$
\boldsymbol{\epsilon}_{n+1}^{e \text { trial }}=\ln \left[\mathbf{U}_{n+1}^{e}{ }_{n+i a l}\right]
$$

where $\mathbf{U}_{n+1}^{e}{ }_{n+1}^{\text {trial }}$ results from polar decomposition of $\mathbf{F}_{n+1}^{e \text { trial }}$ defined by:

$$
\mathbf{F}_{n+1}^{e \text { trial }}=\mathbf{F}_{n+1}\left(\mathbf{F}_{n}^{p}\right)^{-1}
$$

From Eq.(32) we have:

$$
\operatorname{dev}\left(\mathbf{T}_{n+1}\right)=2 \mu \operatorname{dev}\left(\boldsymbol{\epsilon}_{n+1}^{e}\right)
$$

Using Eq.(34), Eq.(35) and Eq.(38) we obtain:

$$
\operatorname{dev}\left(\mathbf{T}_{n+1}\right)=\operatorname{dev}\left(\mathbf{T}_{n+1}^{\text {trial }}\right)-3 \mu \Delta \gamma \frac{\operatorname{dev}\left(\mathbf{T}_{n+1}\right)}{J_{2}\left(\mathbf{T}_{n+1}\right)}
$$

with:

which leads to:

$$
\mathbf{T}_{n+1}^{\text {trial }}=\mathbf{C}^{e}: \boldsymbol{\epsilon}_{n+1}^{e \text { trial }}
$$

$$
J_{2}\left(\mathbf{T}_{n+1}\right)=J_{2}\left(\mathbf{T}_{n+1}^{\text {trial }}\right)-3 \mu \Delta \gamma
$$

We assume that $f_{y_{n+1}}^{\text {trial }}=J_{2}\left(\mathbf{T}_{n+1}^{\text {trial }}\right)-\sigma_{y}(\Delta \gamma)$ is a non-linear scalar function. In the case of a plastic increment $\left(f_{y_{n+1}}^{\text {trial }}>0\right)$, we must solve for $\Delta \gamma$ :

$$
J_{2}\left(\mathbf{T}_{n+1}^{\text {trial }}\right)-3 \mu \Delta \gamma-\sigma_{y}(\Delta \gamma)=0 \leftrightarrow J_{2}\left(\mathbf{T}_{n+1}\right)-\sigma_{y}(\Delta \gamma)=0
$$

A classical Newton-Raphson procedure has been used in this work to solve the above equation.

Using Eq.(39) we obtain:

$$
\operatorname{dev}\left(\mathbf{T}_{n+1}\right)=\frac{\operatorname{dev}\left(\mathbf{T}_{n+1}^{\text {trial }}\right)}{1+\frac{3 \mu \gamma}{J_{2}\left(\mathbf{T}_{n+1}\right)}}
$$

with $J_{2}\left(\mathbf{T}_{n+1}\right)$ defined accorind to Eq.(41) and:

$$
\mathbf{T}_{n+1}=\operatorname{dev}\left(\mathbf{T}_{n+1}\right)+\frac{1}{3} \operatorname{Tr}\left(\mathbf{T}_{n+1}^{\text {trial }}\right)
$$


The Cauchy stress tensor may thus be obtained as:

$$
\boldsymbol{\sigma}_{n+1}=\frac{1}{\operatorname{det}\left(\mathbf{F}_{n+1}\right)}\left[\mathbf{R}_{n+1}^{\text {etrial }}\right]^{-T} \mathbf{T}_{n+1}\left[\mathbf{R}_{n+1}^{\text {etrial }}\right]^{-1}
$$

Finally using Eq.(34) and Eq.(36), and assuming that:

$$
\mathbf{R}_{n+1}^{e}=\mathbf{R}_{n+1}^{e \text { trial }}
$$

we obtain, after some calculations, the following incremental law for $\mathbf{F}^{p}$ (see $[104,105,106]$ ):

$$
\mathbf{F}_{n+1}^{p}=\exp \left[\Delta \gamma \mathbf{N}_{n+1}\right] \mathbf{F}_{n}^{p}
$$

As noticed in [103], as a consequence of the exponential mapping in the implicit integration of the plastic flow rule, the incompressibility of the plastic flow for pressure insensitive yield criteria is carried over exactly to the incremental rule (47). The algorithm, therefore, generalizes the standard return mapping algorithms [108] of the infinitesimal theory. The overall algorithm for the incremental stress update is outlined in Box 2.

(i) For given displacement $\mathbf{u}^{n+1}$, evaluate total deformation gradient $\mathbf{F}^{n+1}=\mathbf{1}+\nabla_{X} \mathbf{u}^{n+1}=\mathbf{1}+\mathbf{B}(\mathbf{X}) \mathbf{u}^{n+1}$

with $\mathbf{B}(\mathbf{X})$ a matrix containing the shape functions derivatives in the reference configuration.

(ii) Evaluate elastic trial deformation gradient

$$
\left(\mathbf{F}^{e}\right)_{n+1}^{\text {trial }}=\left(\mathbf{F}_{n+1}\right)\left(\mathbf{F}_{n}^{p}\right)^{-1}
$$

(iii) Perform polar decomposition of $\left(\mathbf{F}^{e}\right)_{n+1}^{\text {trial }}$

$\left(\mathbf{F}^{e}\right)_{n+1}^{\text {trial }}=\mathbf{R}_{n+1}^{e} \mathbf{U}_{n+1}^{e \text { trial }}$

(iv) Evaluate elastic trial logarithmic strain tensor

$$
\left(\boldsymbol{\epsilon}^{e}\right)_{n+1}^{\text {trial }}=\ln \left[\mathbf{U}_{n+1}^{e \text { trial }}\right]
$$

(v) Evaluate trial stress tensor:

$\mathbf{T}_{n+1}^{\text {trial }}:=\mathbf{C}^{e}:\left(\boldsymbol{\epsilon}_{n+1}^{e}\right)^{\text {trial }}$

(vi) Check plastic consistency condition

IF $J_{2}\left(\mathbf{T}_{n+1}^{\text {trial }}\right)-\sigma_{y}^{n} \leq 0$ THEN :

$(.)_{n+1}=(.)_{n+1}^{\text {trial }}$ and RETURN

ELSE go to (vii)

(vii) Plastic corrector (solve for $\Delta \gamma$ )

$J_{2}\left(\mathbf{T}_{n+1}\right)-\sigma_{y}^{n+1}(\Delta \gamma)=0$, with $J_{2}\left(\mathbf{T}_{n+1}\right)=J_{2}\left(\mathbf{T}_{n+1}^{\text {trial }}\right)-3 \mu \Delta \gamma$

(viii) Update Cauchy stress tensor

$$
\begin{aligned}
& \boldsymbol{\sigma}_{n+1}=J_{n+1}^{-1}\left[\mathbf{R}_{n+1}^{e}\right]^{-T} \mathbf{T}_{n+1} \mathbf{R}_{n+1}^{e-1}, \text { with } \mathbf{T}_{n+1}=\operatorname{dev}\left(\mathbf{T}_{n+1}\right)+\frac{1}{3} \operatorname{Tr}\left(\mathbf{T}_{n+1}^{\text {trial }}\right) \mathbf{1} \\
& \operatorname{dev}\left[\mathbf{T}_{n+1}\right]=\frac{\operatorname{dev}\left[\mathbf{T}_{n+1}^{\text {trial }}\right]}{1+\Xi}, \Xi=\frac{3 \mu \Delta \gamma}{J_{2}\left(\mathbf{T}_{n+1}\right)}
\end{aligned}
$$

(ix) Update plastic part of deformation gradient $\mathbf{F}_{n+1}^{p}=\exp \left[\Delta \gamma \mathbf{N}^{n+1}\right] \mathbf{F}_{n}^{p}, \quad \mathbf{N}^{n+1}=\frac{3}{2} \frac{\operatorname{dev}\left(\mathbf{T}_{n+1}\right)}{J_{2}\left(\mathbf{T}_{n+1}\right)}$

Box 2. Algorithm for integration of constitutive equations

\subsubsection{Explicit Lagrangian procedure}

With the principle of virtual work as a basis of kinematically based C-NEM solution scheme, the corresponding continuum incremental boundary value problem is formulated in the 
spatial configuration as follows

$$
\int_{\Omega^{t}} \rho(t) \ddot{\mathbf{u}} \cdot \boldsymbol{\eta} d \Omega^{t}+\int_{\Omega^{t}} \boldsymbol{\sigma}^{t}: \nabla_{\mathbf{x}} \boldsymbol{\eta} d \Omega^{t}=\int_{\Omega^{t}} \rho(t) \mathbf{b} \cdot \boldsymbol{\eta} d \Omega^{t}+\int_{\partial \Omega_{\sigma}^{t}} \tau \cdot \boldsymbol{\eta} d \Gamma^{t} \forall \eta \in \vartheta
$$

where $\rho$ is the density, $\mathbf{b}$ and $\tau$ represent the body forces and applied tractions respectively, and $\vartheta$ is the space of virtual displacements. The properties $d \Omega^{t}=J^{t} d \Omega_{0}$ and $\rho_{0} d \Omega_{0}=$ $\rho(t) d \Omega^{t}$ are used, which leads to:

$$
\int_{\Omega_{0}} \rho_{0} \ddot{\mathbf{u}} \cdot \boldsymbol{\eta} d \Omega_{0}+\int_{\Omega_{0}} \mathbf{P}^{t}: \nabla \mathbf{x} \boldsymbol{\eta} d \Omega_{0}=\int_{\Omega_{0}} \rho_{0} \mathbf{b} \cdot \boldsymbol{\eta} d \Omega_{0}+\int_{\partial \Omega_{\sigma}^{t}} \tau \cdot \boldsymbol{\eta} d \Gamma^{t} \forall \eta \in \vartheta
$$

where $\mathbf{P}$ denotes the first Piola-Kirschhoff stress tensor related to $\boldsymbol{\sigma}$ by $\mathbf{P}=J \mathbf{F}^{-1} \boldsymbol{\sigma}$.

The C-NEM discretization (71) of the variational form (49) results in the discrete set of algebraic time dependent equations which may be expressed, in matrix form, as:

$$
\mathbf{M} \ddot{\mathbf{u}}_{n+1}(t)=\mathbf{F}_{n}^{\text {ext }}(t)-\mathbf{F}_{n}^{\text {int }}\left(\mathbf{u}_{n}, t\right)
$$

where $t$ is the time, $\mathbf{M}$ denotes the mass matrix, $\mathbf{F}_{n}^{\text {int }}(\mathbf{u}, t)$ the internal force vector, while $\mathbf{F}_{n}^{e x t}(t)$ is the external force vector, expressed, respectively, by:

$$
\begin{gathered}
\mathbf{M}=\int_{\Omega_{0}} \rho_{0} \phi^{T}(\mathbf{X}) \phi(\mathbf{X}) d \Omega_{0} \\
\mathbf{F}_{n}^{i n t}\left(\mathbf{u}_{n}, t\right)=\int_{\Omega_{0}} J_{n} \mathbf{F}_{n}^{-1} \sigma_{n} \mathbf{B}(\mathbf{X}) d \Omega_{0} \\
\mathbf{F}_{n}^{e x t}=\int_{\Omega_{0}} \rho_{0} \phi^{T}(\mathbf{X}) \mathbf{b} d \Omega_{0}+\int_{\partial \Omega_{\sigma}^{t}} \phi^{T}(\mathbf{x}) \tau d \Gamma^{t}
\end{gathered}
$$

with $\phi(\mathbf{X})$ a matrix containing the shape functions in the reference configuration and $\mathbf{B}(\mathbf{X})$ a matrix containing the shape functions derivatives also in the reference configuration. In the following, body forces have been neglected. As shown in the next section, the use of the SCNI quadrature [109] results in a $\mathbf{M}$ matrix diagonal, whose diagonal terms are given by $m_{i}=\rho_{0} \Omega_{i}$, with $\Omega_{i}$ the area (volume in 3D) of the Voronoi cell related to node $n_{i}$.

The velocity $\mathbf{v}=\dot{\mathbf{u}}$ and acceleration $\ddot{\mathbf{u}}=\dot{\mathbf{v}}$ are approximated by using central differences with variable time steps. Thus, we have:

$$
\begin{gathered}
\mathbf{v}_{n+1 / 2}=\mathbf{v}_{n-1 / 2}+\frac{\Delta t_{1}+\Delta t_{2}}{2} \ddot{\mathbf{u}}_{n} \\
\mathbf{u}_{n+1}=\mathbf{u}_{n}+\Delta t_{2} \mathbf{v}_{n+1 / 2}
\end{gathered}
$$

Finally, the general explicit algorithm is outlined as follows. Being known the initial conditions or the computed solution at time: $t_{n}: \mathbf{u}_{n}, \mathbf{v}_{n-1 / 2}, \ddot{\mathbf{u}}_{n}, \mathbf{F}_{n}, \mathbf{F}_{n}^{p}$ :

(i) Update displacements and velocity using Eqs. (54) and (55).

(ii) Evaluate $\boldsymbol{\sigma}_{n+1}, \mathbf{F}_{n+1}^{p}$ by using Box 2 .

(iii) Update accelerations $\ddot{\mathbf{u}}_{n+1}$

$$
\left(\ddot{u}_{i}\right)_{n+1}=\frac{1}{m_{i}}\left\{\left(F_{i}^{e x t}\right)_{n+1}-\left[F_{i}^{i n t}\right]_{n+1}\right\}
$$

Remark: In case of contact, $\ddot{\mathbf{u}}_{n+1}$ is only used as a predictor phase, which has to be corrected according to the prescribed displacements or traction. 


\subsubsection{Thermo-mechanical coupling}

The weak form of the heat balance can be expressed as

$$
\int_{\Omega^{t}} \rho(t) c(t) \dot{T} \eta d \Omega^{t}+\int_{\Omega^{t}} k(t) \nabla_{\mathbf{x}} \mathbf{T} \cdot \nabla_{\mathbf{x}} \eta d \Omega^{t}=\int_{\Omega^{t}} r^{t} \eta d \Omega^{t}+\int_{\partial_{2} \Omega} \bar{q} \eta d \Gamma^{t} \forall \eta \in V_{T}
$$

where $c(t)$ is the specific heat, $k(t)$ is the thermal conductivity for isotropic conduction, and $r^{t}$ a heat source related to the inelastic deformations, defined at time $t$. In the following, we assume $c$ and $k$ constant in time. $V_{T}$ is the space of virtual temperatures and $\bar{q}$ represents the heat transfer at the boundary $\partial_{2} \Omega$. Following similar arguments as in the mechanical problem (48), the weak form of the heat balance can be expressed in the reference configuration by

$$
\begin{array}{rl}
\int_{\Omega_{0}} \rho_{0} c \dot{\mathbf{T}} \eta d \Omega_{0}+\int_{\Omega_{0}} & k \nabla_{\mathbf{X}} \mathbf{T F}^{-T} \mathbf{F}^{-1} \nabla \mathbf{X} \eta d \Omega_{0}=\int_{\Omega_{0}} \operatorname{det}(\mathbf{F}) r^{t} \eta d \Omega_{0} \\
& +\int_{\partial_{2} \Omega} \bar{q} \eta d \Gamma^{t} \forall \eta \in V_{T}
\end{array}
$$

The C-NEM discretization of Eq.(57) results in the ODE system:

$$
\mathbf{C} \dot{\mathbf{T}}+\mathbf{K T}=\mathbf{Q}
$$

which in the context of an explicit scheme can be written as

$$
\mathbf{C T}^{n+1}=[\mathbf{C}+\Delta t \mathbf{K}] \mathbf{T}^{n}+\Delta t \mathbf{Q}^{n}
$$

with

$$
\begin{gathered}
\mathbf{C}=\int_{\Omega_{0}} \rho_{0} c \boldsymbol{\phi}^{T}(\mathbf{X}) \boldsymbol{\phi}(\mathbf{X}) d \Omega_{0} \\
\mathbf{K}=\int_{\Omega_{0}} k \mathbf{B}^{T}(\mathbf{X}) \mathbf{F}^{-T} \mathbf{F}^{-1} \mathbf{B}(\mathbf{X}) d \Omega_{0} \\
\mathbf{Q}^{n}=\int_{\partial \Omega_{2}^{t}} \phi^{T}(\mathbf{x}) \bar{q} d \Gamma^{t}+\int_{\Omega_{0}} \operatorname{det}(\mathbf{F}) r^{t} \boldsymbol{\phi}(\mathbf{X}) d \Omega_{0}
\end{gathered}
$$

The matrix $\mathbf{C}$ becomes diagonal in the context of C-NEM when stabilized conforming nodal integration is used, being the diagonal terms $c_{i}=\rho_{0} c \Omega_{i}$. The heat source resulting from the inelastic deformations is given by

$$
r^{t}=\chi \boldsymbol{\sigma}^{t}: \hat{\mathbf{D}}_{t}^{p}
$$

with

$$
\hat{\mathbf{D}}_{t}^{p}=\operatorname{sym}\left[\left(\dot{\mathbf{F}}_{t}^{p}\right)\left(\mathbf{F}_{t}^{p}\right)^{-1}\right]
$$

where $\boldsymbol{\sigma}^{t}$ is the Cauchy stress tensor et time $t$, and $\chi$ is the Taylor-Quinney parameter [110] representing the fraction of plastic work converted into heat. In the present work, we have used $\chi=0.9$ [69]. The thermo-mechanical coupling is carried out by the effects of inelastic deformations (62) and by the softening effects due to temperature in the hardening law. The hardening law adopted in the present simulation is given by the classical Johnson-Cook law $[56]$

$$
\sigma_{y}\left(\bar{\epsilon}^{p}\right)=\left[A+B\left(\bar{\epsilon}^{p}\right)^{n}\right]\left[1+C \ln \left(\frac{\dot{\epsilon}^{p}}{\bar{\epsilon}_{0}^{p}}\right)\right]\left[1-\left(\frac{T-T_{0}}{T_{f}-T_{0}}\right)^{m}\right]
$$

where $A, B, C, m, n$ and $\dot{\epsilon}_{0}^{p}$ are material parameters, $\bar{\epsilon}^{p}$ represents the equivalent plastic strain and $\dot{\bar{\epsilon}}^{p}$ represents the rate of plastic strain. $T$ and $T_{0}$ correspond to the current and initial temperature respectively. 
When mechanical and thermal problems are coupled, a staggered solution approach is usually adopted, which solves the mechanical and the thermal problems in an uncoupled manner with data exchange performed at the end of each time step or increment. In particular, the nodal temperatures are transferred to the mechanical procedure, while plastic work is communicated to the thermal solver.

In order to handle large strains or adaptive refinement easily, an appropriate discretization scheme must be applied to the above constitutive equations. For this purpose, the interest of meshfree methods is presented below.

\subsection{Meshfree Approaches}

Meshless methods have been developed over the past decade to circumvent the inherent difficulties of the traditional finite element method. Different methods can be found, such as the diffuse element method (DEM) [111], the element free Galerkin method (EFG) [112], the reproducing kernel particle method [113], SPH and radial basis functions methods $[114,115]$, or the natural element method (NEM) [116].

Some advantages of meshfree methods over mesh-based techniques are: (a) the possibility to handle geometric changes like free surfaces or large deformation better, as the quality of the approximation is less sensible to the relative position of the nodes, and due to the larger connectivity [117]; (b) the generation of the cloud of nodes is not constraint by a criterion (i.e. minimum angle) on the relative position of the nodes, which simplifies the task in the 3D case [118]; (d) the possibility to add or remove freely some nodes for adaptivity purpose; (e) the possibility to define the internal variables at the nodes, which removes the numerical diffusion in the case of projection of the fields between updates [119];

On the other hand, the following drawbacks are usually attributed to meshfree methods: (a) issues related to boundary conditions $[112,120]$; (b) issues related to numerical integration [121]; (c) the size of the shape functions (domain of influence) is in the vast majority of meshless methods radial, and a size parameter must be appropriately chosen to avoid instability or loss of accuracy [113, 122]; (d) finally, some difficulties in the treatment of non-convex domains and material interfaces [123] occur.

The so-called constrained natural element method (C-NEM) [124] is a recent meshfree methods, in which most of the drawbacks founds in most meshfree methods are alleviated. Basic theory about the method and its interest in machining simulation is described as follows.

\subsection{The C-NEM Approach}

\subsubsection{Natural neighbor interpolation}

We briefly touch upon the foundation of Sibson's natural neighbor coordinates (shape functions) that are used in the natural element method. For a more in-depth discussion on the Sibson interpolant and its application for solving second-order partial differential equations, the interested reader can refer to Braun and Sambridge [125], and Sukumar et al. [116]. The NEM interpolant is constructed on the basis of the Voronoi diagram. The Delaunay tessellation is the topological dual of the Voronoi diagram.

Consider a set of nodes $S=\left\{n_{1}, n_{2}, \ldots, n_{N}\right\}$ in $\Re^{d i m}$, being dim the space dimension considered. The Voronoi diagram is the subdivision of $\Re^{d i m}$ into regions $T_{i}$ (Voronoi cells) defined by:

$$
T_{i}=\left\{\mathbf{x} \in \Re^{d i m}: d\left(\mathbf{x}, \mathbf{x}_{i}\right)<d\left(\mathbf{x}, \mathbf{x}_{j}\right), \forall j \neq i\right\}, \quad \forall i
$$

The Sibson coordinates of $\mathbf{x}$ with respect to a natural neighbor $n_{i}$ (see Figure 11) is defined as the ratio of the overlap area (volume in $3 \mathrm{D}$ ) of their Voronoi cells to the total 


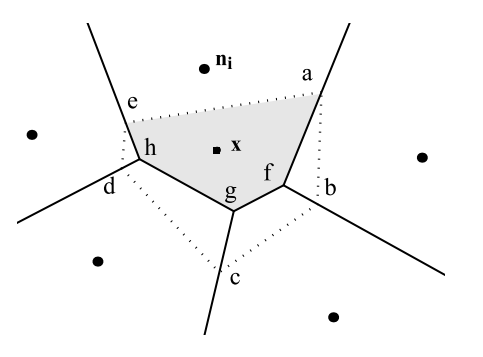

(a)

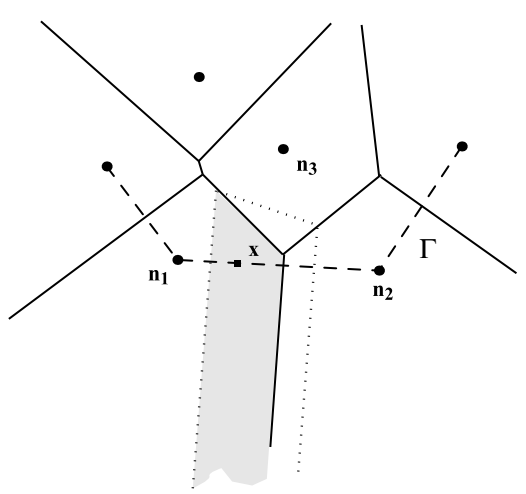

(b)

Figure 11. Construction of the Sibson shape functions

area (volume in 3D) of the Voronoi cell related to point $\mathbf{x}$ :

$$
\phi_{i}(\mathbf{x})=\frac{\text { Area }(\text { afghe })}{\text { Area }(\text { abcde })}
$$

If the point $\mathbf{x}$ coincides with the node $n_{i}$, i.e. $\left(\mathbf{x}=\mathbf{x}_{i}\right), \phi_{i}\left(\mathbf{x}_{i}\right)=1$, and all other shape functions are zero, i.e. $\phi_{i}\left(\mathbf{x}_{j}\right)=\delta_{i j}\left(\delta_{i j}\right.$ being the Kronecker delta). The properties of positivity, interpolation, and partition of unity are then verified [116]:

$$
\left\{\begin{array}{l}
0 \leq \phi_{i}(\mathbf{x}) \leq 1 \\
\phi_{i}\left(\mathbf{x}_{j}\right)=\delta_{i j} \\
\sum_{i=1}^{n} \phi_{i}(\mathbf{x})=1
\end{array}\right.
$$

The natural neighbor shape functions also satisfy the local coordinate property [126], namely:

$$
\mathbf{x}=\sum_{i=1}^{n} \phi_{i}(\mathbf{x}) \mathbf{x}_{i}
$$

which combined with Eq.(67) implies that the natural neighbor interpolant spans the space of linear polynomials (linear completeness).

It turns out that the support of $\phi_{i}(\mathbf{x})$ is the union of the $n$ circles (spheres in 3D) passing through the vertices of the $n$ Delaunay triangles (tetrahedra) containing the node $n_{i}$ (in this case $n$ is the number of natural neighbors of node $n_{i}$ ). The support of a node $n_{i}$ in a particular nodal distribution is depicted in Figure 12.

Natural neighbor shape functions are $C^{\infty}$ at any point except at the nodes, where they are only $C^{0}$, and on the boundary of the Delaunay circles (spheres in 3D) where they are only $C^{1}$, because of the discontinuity in the neighbors nodes across these boundaries. Hiyoshi and Sugihara [127] have shown that the Sibson interpolant belongs to a more general class of Voronoi-based interpolants, called $k$-th order standard coordinates, proving that the interpolant generated by the k-th order standard coordinates have $C^{k}$ continuity on the Delaunay circles (spheres) boundaries. In this context, the Sibsonian and nonSibsonian (Laplace) coordinates [128] results to be the standard coordinates of order 1 and 0 , respectively.

Another important property of this interpolant is the ability to reproduce linear functions over the boundary of convex domains. The proof can be found in Sukumar et al. 


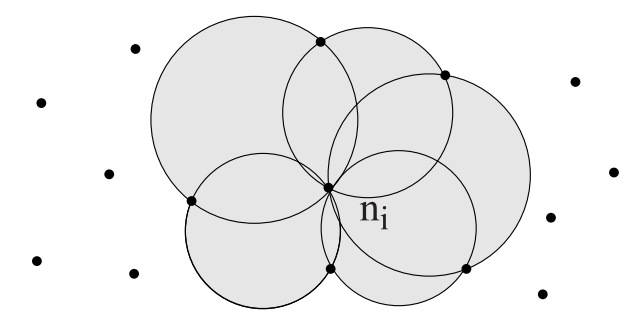

Figure 12. Support of the natural shape function related to node $\mathbf{n}_{i}$

[116]. An illustration is depicted in Figure 11 (b): as the areas associated to points on the boundary become infinite, the contribution of internal points vanish in the limit when the point approaches the convex boundary, and the shape functions associated with nodes $n_{1}$ and $n_{2}$ become linear on the segment $\left(n_{1}-n_{2}\right)$. This is not true in the case of non convex boundaries, and the next section focuses on an approach to circumvent this difficulty.

Consider an interpolation scheme for a function (vectorial, scalar or tensorial) $\mathbf{u}(\mathbf{x})$, in the form:

$$
\mathbf{u}^{h}(\mathbf{x})=\sum_{i=1}^{n} \phi_{i}(\mathbf{x}) \mathbf{u}_{i}
$$

where $\mathbf{u}_{i}$ are the nodal values at the $n$ natural neighbor nodes, and $\phi_{i}(\mathbf{x})$ are the shape functions associated with each neighbor node. It is noted that Eq.(69) defines a local interpolation scheme. Thus, the trial and test functions used in the discretization of the variational formulation describing both the mechanical and thermal problems treated in this paper take the form of Eq.(69).

\subsubsection{The Constrained natural element method}

\section{Constrained Voronoi diagram}

In its original form [116], the NEM can only be applied to strictly convex domains. For non-convex domains, two main issues occur: (a) It was proved in [116, 124] and [129] that a loss of linearity in the interpolation along boundaries of non convex domains appear. Thus essential boundary conditions can only be imposed directly over convex boundaries; (b) For strongly non-convex domains (cracks, auto-contact...) some spurious influences between nodes of the boundaries appear [124]. An additional treatment is thus required to maintain all the properties of the NEM for any geometry.

In order to avoid these drawbacks, we have proposed in a previous paper [124] an extension of the NEM in which a visibility criterion is introduced in order to restrict influent nodes among natural neighbors. The computation of the shape functions is done on the basis of the so-called constrained (or extended) Voronoi diagram (CVD), which is the strict dual to the constrained Delaunay triangulation, introduced by Seidel in [130], instead of the Voronoi diagram (see [124] for further details). The intersection between the CVD and the domain results into new cells $T_{i}^{C}$, called constrained Voronoi cells, defined formally by:

$$
T_{i}^{C}=\left\{\mathbf{x} \in \Re^{n}: d\left(\mathbf{x}, \mathbf{x}_{i}\right)<d\left(\mathbf{x}, \mathbf{x}_{j}\right), \quad \forall j \neq i, S_{x \rightarrow n_{i}} \cap \Gamma=\emptyset, S_{x \rightarrow n_{j}} \cap \Gamma=\emptyset\right.
$$

where $\Gamma$ is the domain boundary, composed by a set of segments $l_{i} \in L$ and $S_{a \rightarrow b}$ denotes the segment between the points $a$ and $b$. In this framework, a point located inside a cell $T_{i}^{C}$ is closer to the node $n_{i}$ than to any other visible node $n_{j}$.

The constrained Delaunay triangulation does not always exist in $3 \mathrm{D}$ without adding new nodes, as shown in [131]. Nevertheless, some techniques for constructing 3D constrained Delaunay tessellations are available and provided in $[132,133]$ by adding Steiner points. 
The constrained natural element approximation

In order to solve partial differential equations defined on non convex domains, or to reproduce functional discontinuities, we consider the following approximation for both the trial and the test functions:

$$
\mathbf{u}^{h}(\mathbf{x})=\sum_{i=1}^{V} \phi_{i}^{C}(\mathbf{x}) \mathbf{u}_{i}
$$

where $V$ is the number of natural neighbors visible from point $\mathbf{x}$ and $\phi_{i}^{C}$ is the constrained natural neighbor shape function related to the $\mathrm{i}$-th node at point $\mathbf{x}$. The computation of the C-n-n (constrained natural neighbor) shape functions is similar to the natural neighbor shape function, when one proceed using the constrained Voronoi diagram introduced previously. It was shown in [124] and [134] that the use of the constrained Voronoi diagram does not affect the properties of the NEM interpolation, allowing the extension of the linearity of the shape functions to any geometry, convex or not.

The ability of the C-NEM for treating problems involving cracks has been illustrated in [124] and in the context of moving interfaces in [134], where we have shown how the C-NEM simplifies the treatment of material discontinuities in meshfree methods, due to the continuity of the approximation across interfaces (consequence from the interpolant character and linearity of the C-NEM shape functions over any external boundary or internal interface). It is noteworthy that no size parameter is involved in the definition of the influent nodes (neighbors), which is essential for the robustness of refinement procedures, in which the nodal density varies in some parts of the domain.

\subsection{First Result with the C-NEM}

Some features of the C-NEM, such as the possibility to handle large transformations of the domain without remeshing (nodal repositioning) and without field projection, to easily described cracks or discontinuities, the direct imposition of essential boundary conditions, or the possibility to refine the cloud of nodes without size support parameter, makes the C-NEM an appealing candidate to discretize the constitutive equations for the simulation of machining, among the many other available schemes.

One important point in cutting or blanking simulations is the accurate description of cracks or material interface in domains that can be submitted to large strains. The C-NEM offers a simple framework in that context, as material interfaces can be inserted arbitrarily in the cloud of nodes, without any restriction on the relative position of the nodes. To illustrate the performance of the method to describe the material interfaces of material separation, a simple benchmark involving a cracked domain is presented.

\subsubsection{Crack analysis}

In this example, we consider the problem defined in Figure 13 where $2 a=0.2 \mathrm{~mm}, 2 W=2$ $\mathrm{mm}$ and $\sigma_{22}^{\infty}=1 \mathrm{Mpa}$. Plane stress conditions are assumed with an elastic modulus of $E=1 \mathrm{MPa}$ and a Poisson coefficient of $\nu=0.3$. Firstly, we consider the case of $\gamma=0$ whose exact solution in an infinite plate was obtained by Muskelishvili [135]. For $\gamma=0$ and $\theta=0$ in Figure 13, this solution results:

$$
\left\{\begin{array}{l}
\sigma_{22}(\theta=0, \gamma=0, r)=\sigma_{22}^{\infty} \frac{a+r}{\sqrt{r(2 a+r)}} \\
\sigma_{11}(\theta=0, \gamma=0, r)=\sigma_{22}^{\infty}-\sigma_{22}
\end{array}\right.
$$

When the crack size is much smaller than the domain size, Muskelishvili's solution can be taken as a reference solution.

A cloud of 760 nodes is considered in the C-NEM simulation (400 nodes are uniformly distributed, other 20 nodes are located on each crack side and the remaining 320 nodes 

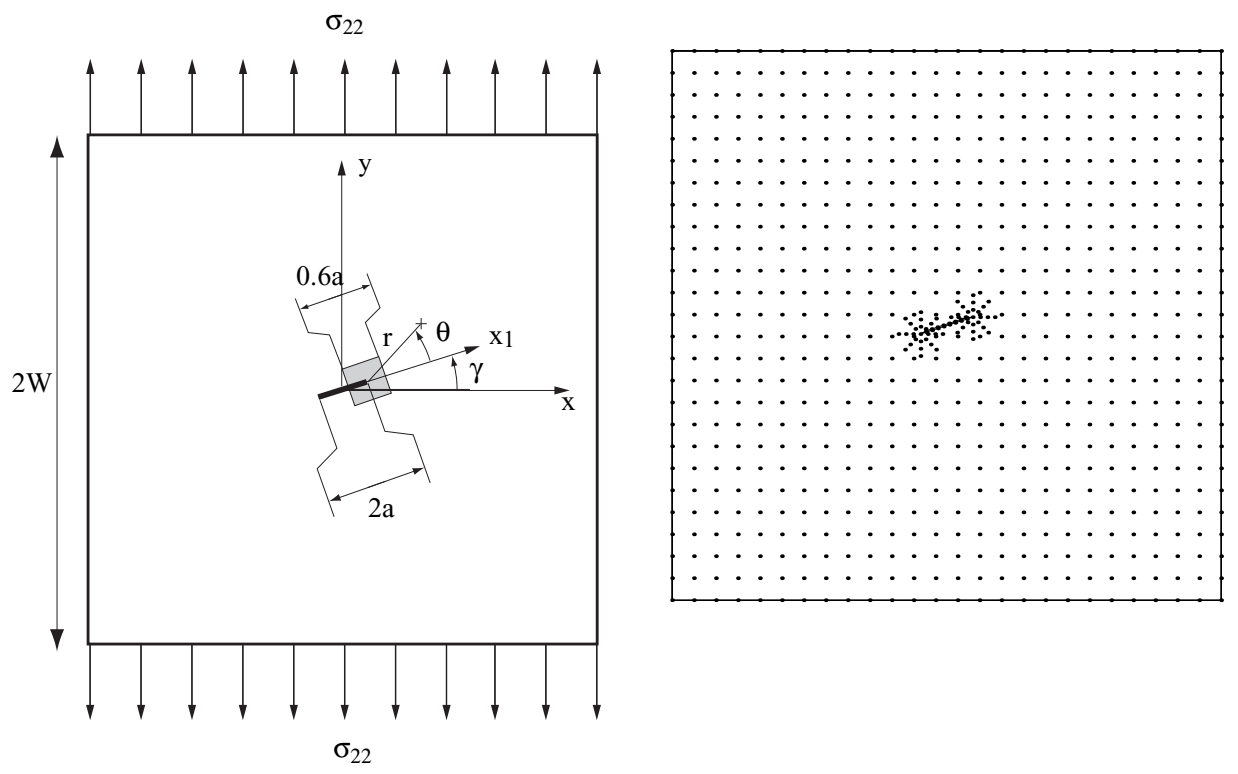

Figure 13. Square plate with an inclined crack.(a) Geometry and loads. (b) Distribution of nodes

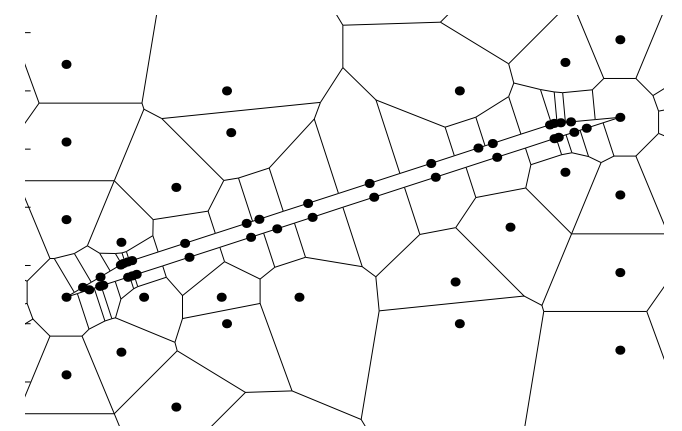

Figure 14. Zoom of the constrained Voronoi diagram in the neighborhood of the crack (detail)

are concentrated in the crack tip zones) as depicted in Figure 13 without assuming any symmetry condition. Numerical results are reported in Figure 15.

Now, we consider the case of an inclined crack. The stress intensity factor (SIF) $K_{I}$ has been computed using the contour form of the interaction integral method [136], the different values of interest being easily interpolated anywhere in the domain. The size of the square domain used in the computation is $0.6 a \times 0.6 a$ (see Figure 6). Numerical results concerning the intensity factor $K_{I}$ for different values of $\gamma$ are compared in Figure 16 with the exact ones given in [135]:

$$
K_{I}=\sigma_{22}^{\infty} \sqrt{\pi a} \cos ^{2} \gamma
$$

Accuracy can be improved by increasing the nodal density in the crack tip neighborhood. Another possibility is to extrinsically enhance the trial function by adding terms reproducing the local $\sqrt{r}$ behaviour. As the shape functions $\phi_{i}^{C}(\mathbf{x})$ define a partition of unity, the enrichment of the C-NEM trial function in crack problems can be achieved in the partition of unity framework [137]. Let $\Omega_{0} \subset \Omega$ be a sub-domain in a region around the crack-tip 


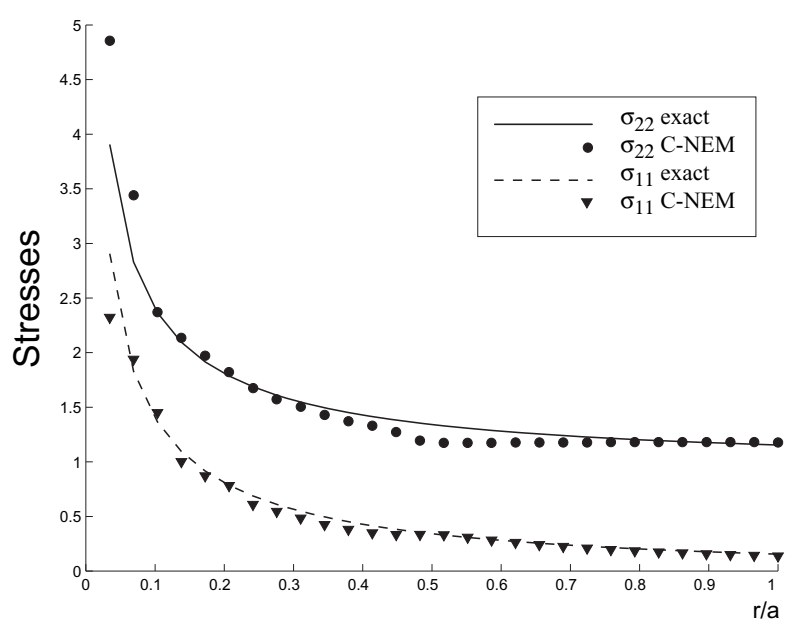

Figure 15. Radial stresses ahead of the crack tip for $\gamma=0$

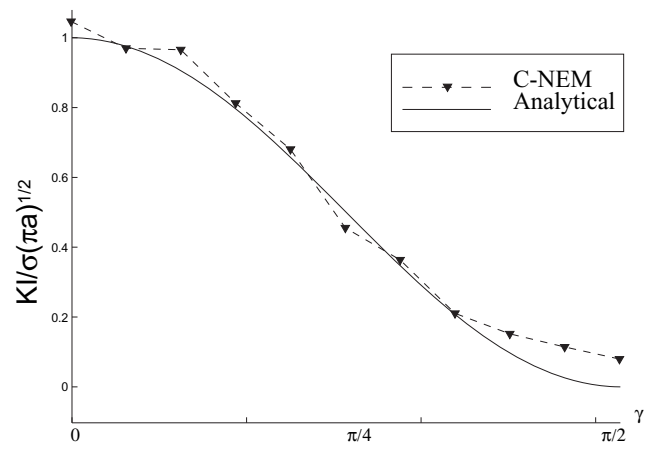

Figure 16. Stress intensity factor $K_{I}$ for diferent values of the crack orientation $\gamma$

where the crack-tip singularity domainates. Then the enriched function for two-dimensional crack problems could follow the form suggested by Sukumar in [116]:

$$
\mathbf{u}^{h}(\mathbf{x})=\sum_{i=1}^{n} \phi_{i}^{c}(\mathbf{x}) \mathbf{u}_{i}+\sqrt{r} \sum_{j=1}^{m} \phi_{j}^{c}(\mathbf{x}) \mathbf{a}_{j}
$$

where $\mathbf{a}_{j}$ are additional nodal coefficients that are associated with the $m$ nodes in $\Omega_{0}$.

\subsubsection{Adaptive refinement}

In this section, an adaptive strategy is proposed for non-linear problems with historydependent internal variables in the C-NEM context, including: (a) a Zienkiewicz-Zhu error indicator [138] based on equivalent plastic strain; (b) a transfer technique based on the stabilized conforming nodal integration [109] and (c) a refinement strategy for domains evolving in time based on the Voronoi cells.

In the context of the finite element method, the transfer of internal variables between successive remeshing operations is an important issue (see i.e. [139, 140, 103]). In this work, we use the stabilized conforming nodal integration proposed by Chen et al. [109] to define all variables at the nodes, in order to avoid projection between two successive actualisation of the reference configuration (update of the Voronoi diagram and shape functions). 


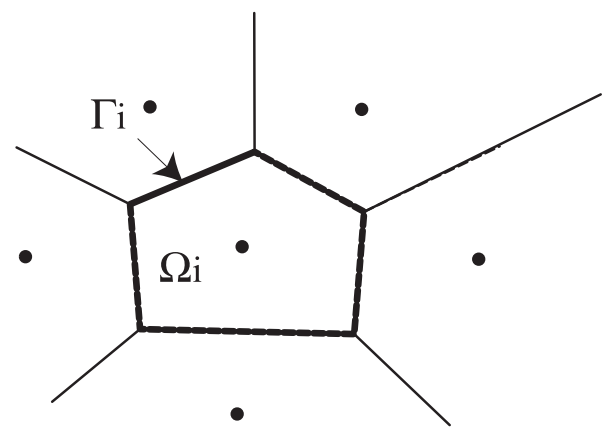

Figure 17. Representative domain around a node for the evaluation of $\tilde{\mathbf{F}}_{i}$

We thus consider a deformation gradient at node $n_{i}$ :

$$
\tilde{\mathbf{F}}_{i}=\frac{1}{\left|\Omega_{i}\right|} \int_{\Omega_{i}}\left(\nabla_{X} u^{h}(\mathbf{x})+\mathbf{1}\right) d \Omega=\mathbf{1}+\tilde{\nabla}_{X} u^{h}\left(\mathbf{x}_{i}\right)=\mathbf{1}+\tilde{\mathbf{B}}_{i} \mathbf{u}_{i}
$$

where $\Omega_{i}$ is a representative domain around the node (typically a Voronoi cell) (see Figure 17). Introducing C-NEM discretization scheme into (75) we obtain:

$$
\tilde{\nabla}_{X} u^{h}\left(\mathbf{x}_{i}\right)=\left[\begin{array}{c}
u_{, X}^{h}\left(\mathbf{x}_{i}\right) \\
u_{Y Y}^{h}\left(\mathbf{x}_{i}\right) \\
v_{, X}^{h}\left(\mathbf{x}_{i}\right) \\
v_{, Y}^{h}\left(\mathbf{x}_{i}\right)
\end{array}\right]=\tilde{\mathbf{B}}_{i} \mathbf{u}_{i}
$$

where $\tilde{\mathbf{B}}_{i}$ is expressed by:

$$
\tilde{\mathbf{B}}_{i}=\left[\begin{array}{ccccccc}
\tilde{\phi}_{1, X} & 0 & \tilde{\phi}_{2, X} & 0 & \ldots & \tilde{\phi}_{N, X} & 0 \\
\tilde{\phi}_{1, Y} & 0 & \tilde{\phi}_{2, Y} & 0 & \ldots & \tilde{\phi}_{N, Y} & 0 \\
0 & \tilde{\phi}_{1, X} & 0 & \tilde{\phi}_{2, X} & \ldots & 0 & \tilde{\phi}_{N, X} \\
0 & \tilde{\phi}_{1, Y} & 0 & \tilde{\phi}_{2, Y} & \ldots & 0 & \tilde{\phi}_{N, Y}
\end{array}\right]
$$

with

$$
\begin{aligned}
\tilde{\phi}_{j, X} & =\frac{1}{\Omega_{i}} \int_{\Omega_{i}} \phi_{j, X}(\mathbf{X}) d \Omega \\
\tilde{\phi}_{j, Y} & =\frac{1}{\Omega_{i}} \int_{\Omega_{i}} \phi_{j, Y}(\mathbf{X}) d \Omega
\end{aligned}
$$

where $\mathbf{u}_{i}$ are the nodal displacements. In the context of a Lagrangian procedure, different options can be considered:

1. A total Lagrangian procedure, where the Voronoi diagram as well as the shape functions are computed only once at the beginning of the simulation;

2. An updated Lagrangian procedure where the Voronoi diagram and the shape functions are adapted every time steps.

3. A periodically updated Lagrangian procedure where the Voronoi diagram and the shape functions are computed after several total Lagrangian steps. 
In the context of a total Lagrangian procedure, no internal variable transfer is necessary. Nevertheless, in updated Lagrangian procedures (2) and (3), it is necessary to update the reference configuration, which implies to reconstruct the new Voronoi diagram associated with the new nodal distribution, as well as the new shape functions. In that case, the use of the assumed gradient defined in (75) allows the definition of all internal variables at the nodes. In the case of an explicit procedure, all quantities defined in the former time step are defined at the nodes in the same way. If the same cloud of nodes is used through the whole simulation, the transfer is thus direct as integration points coincide with the nodes. If a different cloud of node is used during the simulation (due to the refinements or nodal repositioning), a direct C-NEM interpolation (71) can be performed to define the internal variable the new nodes.

In the context of the finite element method, adaptive strategies have been extensively applied and continuously developed for linear [141] [142] [138] and some class of nonlinear problems and history-dependent nonlinear problems over the last two decades or so (see i.e. Ladevèze et al. [143], Belytschko et al. [144], Ortiz et Quigley [145], Gallimard et al. [146], among many others).

The present section aims to propose a simple error indicator for adaptive solutions of large elasto-plastic transformations in the C-NEM framework. In the context of the finite element method, an elementary procedure for the error estimation may be defined by substituting the exact solution by some post-processed field obtained from the available FEM solution. When the finite element solution is accurate enough, the post-processed solution is expected to be more accurate than the original FEM solution. In particular, the a posteriori error estimation procedure originally proposed and used by Zienkiewicz and Zhu [138] for linear elliptic problems is based on the observation that exact stress $\sigma$ may be represented accurately by smoothed stress $\sigma^{*}$ obtained by a suitable projection of stresses $\sigma^{h}$.

In the context of meshless methods, the field $\sigma^{h}$ is smooth in the general case, unlike in the FEM. Many options have been considered to construct error indicators in meshfree methods, mostly using some recovery Zienkiewicz-Zhu fields (see the work by Liu et al. [147], You et al. [148], Chung et Belytschko [149], Lee et Zhou [151, 150] and Lu et Chen [152] for an overview of the recent proposed techniques). In most of the referred papers in which a MLS approximation is used, an important issue is the influence of the shape function support size on the efficiency of error estimates [149]. Furthermore, it has been shown in [150] that adaptive refinement in MLS meshless techniques is a delicate task due to the necessity to adapt locally the shape function support according to the local nodal density. In the following, an error indicator based on the NEM shape functions is proposed to circumvent the just referred difficulties, as the NEM shape functions support automatically adapts its shape and size to the surrounding neighborhood.

We propose a simple error indicator using the Zienckiewicz-Zhu idea in tandem with the stabilized conforming nodal integration. Let $\alpha(\mathbf{X}, t)$ a variable either associated with the spatial derivatives (i.e. $\mathbf{F}(\mathbf{X}, t)$ ), or with internal variables (i.e. $\bar{\epsilon}^{p}(\mathbf{X}, t)$ ). In the C-NEM context, the stabilized conforming nodal integration scheme proposed by Chen et al. [153] produces constant piece-wise fields associated with the different derivatives, discontinuous across the Voronoi cells. This is a consequence of the stabilization scheme used in (75). In addition, the different internal variables can be considered at the nodes, as we have nodal integration. Constant fields $\alpha^{h}\left(\mathbf{X}_{i}, t\right)$ associated with variables $\alpha(\mathbf{X}, t)$ can thus be considered in each Voronoi cell $\Omega_{i}$. A simple solution for recovery fields $\alpha^{*}(\mathbf{X}, t)$ with assumed better accuracy is to interpolate the nodal values of $\alpha^{h}\left(\mathbf{X}_{i}, t\right)$ with the C-NEM 


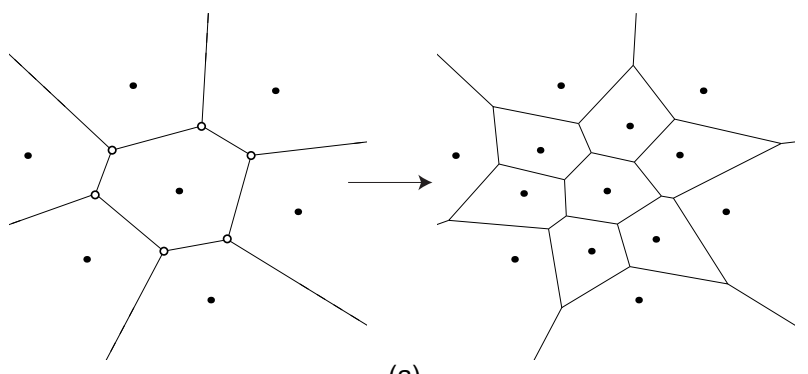

(a)

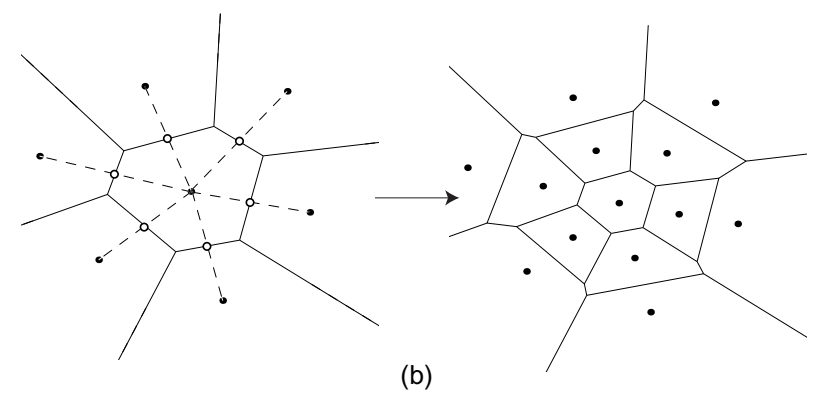

Figure 18. Refinement procedure based on the Voronoi cells. (o): additional nodes

interpolation scheme:

$$
\alpha(\mathbf{X}, t)^{*}=\sum_{i=1}^{V} \phi_{i}^{C}(\mathbf{X}) \alpha^{h}\left(\mathbf{X}_{i}, t\right)
$$

Then, the error based on the equivalent plastic strain for any cell $\Omega_{i}$ may be defined in the reference configuration, as:

$$
|e|_{\Omega_{i}}^{2}=\int_{\Omega_{i 0}}\left(\left[\bar{\epsilon}^{p}(\mathbf{X}, t)\right]^{*}-\left[\bar{\epsilon}^{p}\left(\mathbf{X}_{i}, t\right)\right]^{h}\right)^{2} J_{i} d \Omega_{0}
$$

with

$$
\left[\bar{\epsilon}^{p}(\mathbf{X}, t)\right]^{*}=\sum_{j=1}^{V} \phi_{j}^{c}(\mathbf{X})\left[\bar{\epsilon}^{p}\left(\mathbf{X}_{j}, t\right)\right]^{h} \quad J_{i}=\operatorname{det}\left(\tilde{\mathbf{F}}_{i}\right)
$$

where $\left[\bar{\epsilon}^{p}\left(\mathbf{X}_{j}, t\right)\right]^{h}$ is the equivalent plastic strain associated with the natural neighbor $n_{j}$ of point $\mathbf{x}$, assumed constant in the constrained Voronoi cell $\Omega_{j}$. In order to evaluate (81), the constrained Voronoi cells are triangulated and standard Gauss quadrature is applied on the triangles.

Any kind of error indicators can be constructed on the above framework, i.e. error indicators based on rate of plastic work or based on damage [103]. For the sake of simplicity, we only focus in this study on the error indicator described below. The global error is obtained, in a standard way, by: $|e|_{\Omega}^{2}=\sum_{i}^{N}|e|_{\Omega_{i}}^{2}$. In addition, the relative error $\eta$ is defined as $\eta_{i}:=|e|_{\Omega_{i}} /|e|_{\Omega}$.

A simple refinement strategy can then be used, when the error in a Voronoi cell is above a given tolerence. The adaptation of the nodal density can be performed by adding nodes in the Voronoi cells, between the central node and the neighbors, or on the vertices of the Voronoi cell, as depicted in Figure 18. The details of the preocedure can be found in [119]. 


\subsubsection{Numerical simulation of high-speed shearing with the C-Nem}

High-speed shearing is a new metal cutting operation which offers significant advantages compared to traditional shearing. In this process, the high velocity of the punch induces adiabatic shear bands which combined with the damage phenomena produces high-quality of the cutting zone, without any burr formation. This process allows to perform shearing in high strength material such as titanium, or to produce holes in plates with relatively large thickness (up to $20 \mathrm{~mm}$ ) in traditional metals such as aluminium.

The example shows a first insight into the simulation of a plane-strain, high-speed shearing operation using the error indicator and the refinement procedure described previously. The geometry of the problem is depicted in Figure 19. The following material data for stainless steel 304L have been used: $A=253 \mathrm{MPa}, B=685 \mathrm{MPa}, C=0.0973 n=0.312$, $m=2.044, \dot{\bar{\epsilon}}_{0}^{p}=1 ; T_{0}=296 \mathrm{~K}, T_{f}=1698 \mathrm{~K}, E=210 \mathrm{GPa}, \nu=0.33, c=500 \mathrm{~J} / \mathrm{kgK}$, $k=5.86 \mathrm{~J} / \mathrm{JgmK}, \rho=7850 \mathrm{~kg} / \mathrm{m}^{3}$. The initial velocity of the punch is taken as $\mathrm{V}=11$ $m . s^{-1}$.

The main aim was to illustrate the capability of the refinement procedure to capture the details of the shear bands when different geometries of the tools are used. The high speed of the punch, combined to the material characteristics, induces high strain localization. The high rate of energy generation induced by the plastic deformation and the low thermal diffusivity of the material causes localized heating, which in turn, causes thermal softening and consequently localized plastic deformation. Two test have been performed using the following geometrical parameters: $R=5 \mathrm{~mm}, L=20 \mathrm{~mm}, H=10 \mathrm{~mm}$ and $j=0.2 \mathrm{~mm}$.

In a first test, zero corner radii $R_{1}$ and $R_{2}$ have been used. Figure 20 shows the refined nodal distribution during the shear band formation. Figure 21 illustrates the evolution of the underlying Voronoi diagram used for the refinement procedure in half of the workpiece. Figure 22 depicts the equivalent plastic strain. In this first example, the direction of the shear band is observed parallel to the punch speed direction.

In a second test, corner radii $R_{1}=0.5 \mathrm{~mm}$ and $R_{2}=0.5 \mathrm{~mm}$ have been used. We can notice that the increase in the corner radius induces inclined shear bands, which causes conical deformation in the workpiece and lower quality of the manufactured workpiece.

At least, we show on Figure 23 first results of a 3D simulation with 16, 200 Voronoi cells (ie 48,600 Degrees of Freedom). For the example the initial velocity of the punch is taken as $V=10 \mathrm{~m} . \mathrm{s}^{-1}$ and the material behaviour is identical to the previous $2 \mathrm{D}$ simulations.

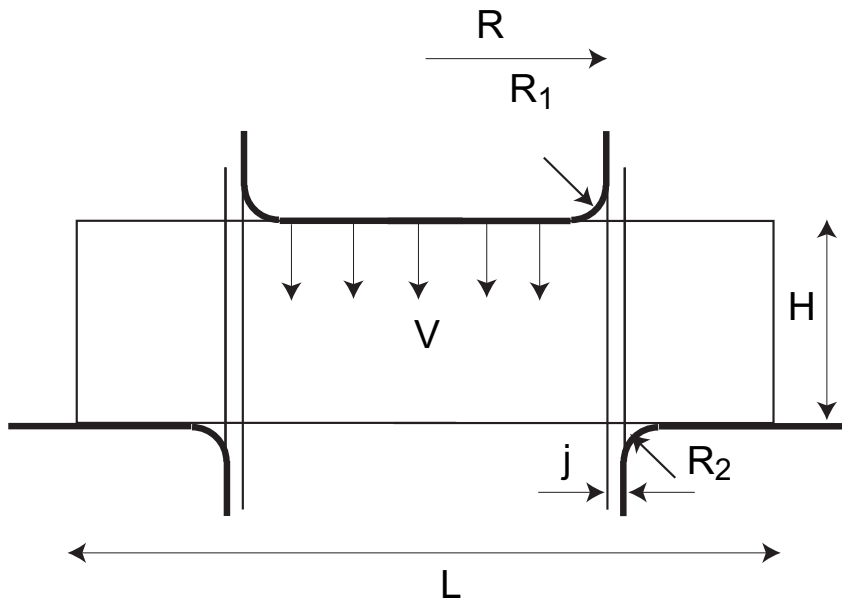

Figure 19. High-speed shearing: geometry of the problem 


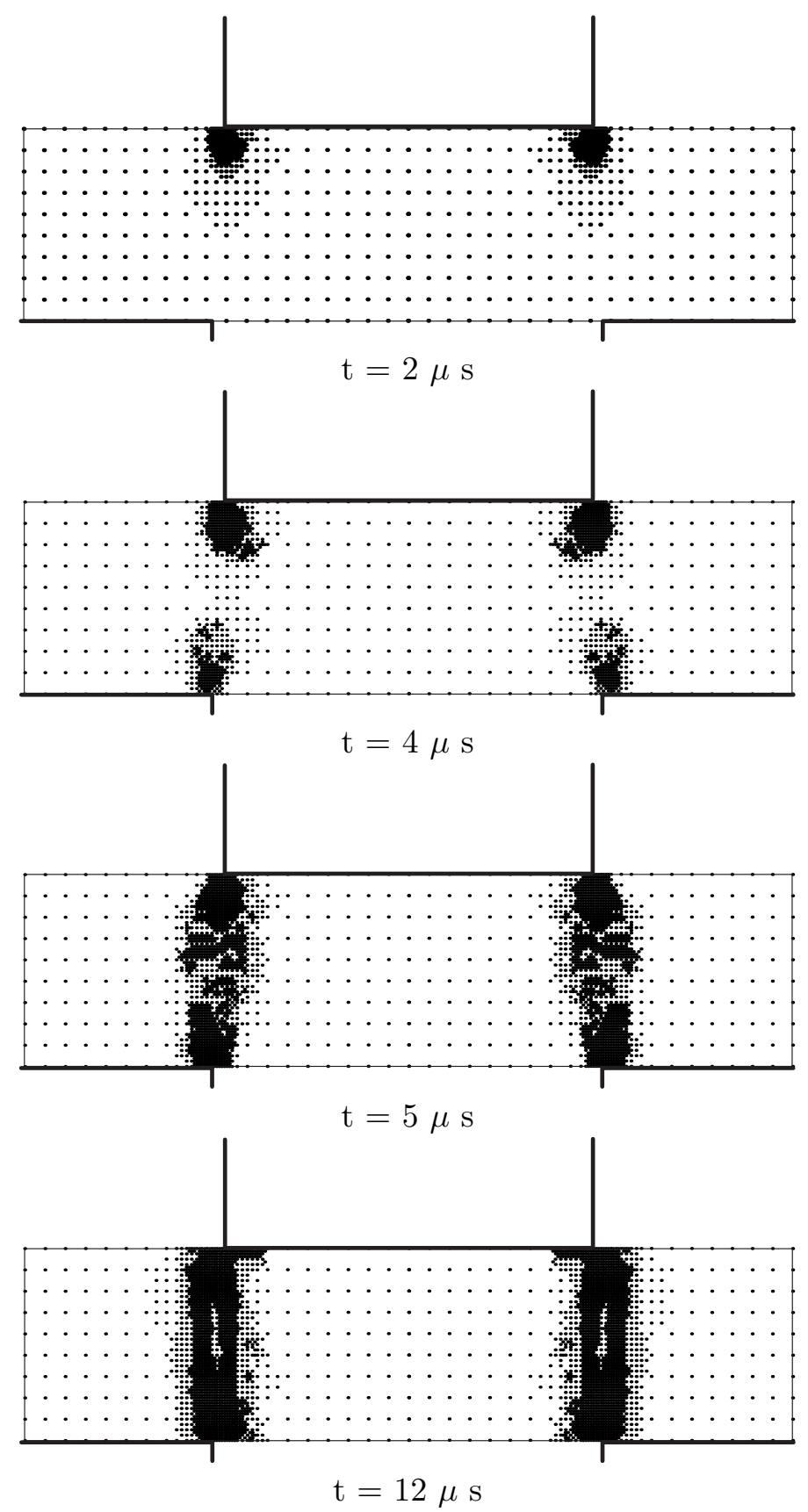

Figure 20. High-speed shearing $\left(R_{1}=0 \mathrm{~mm}\right.$ and $\left.R_{2}=0 \mathrm{~mm}\right)$ : adaptive refinement during shear band formation

\subsubsection{Preliminary results of cutting simulations using the natural element method}

Preliminary results of cutting simulations using the natural element method are shown with 2D simulation in a case of orthogonal cutting on Figure 24. As it can be see the refinement procedure is applied to this example.

On Figure 25 some 3D effects can be see with a 3D simulation. 

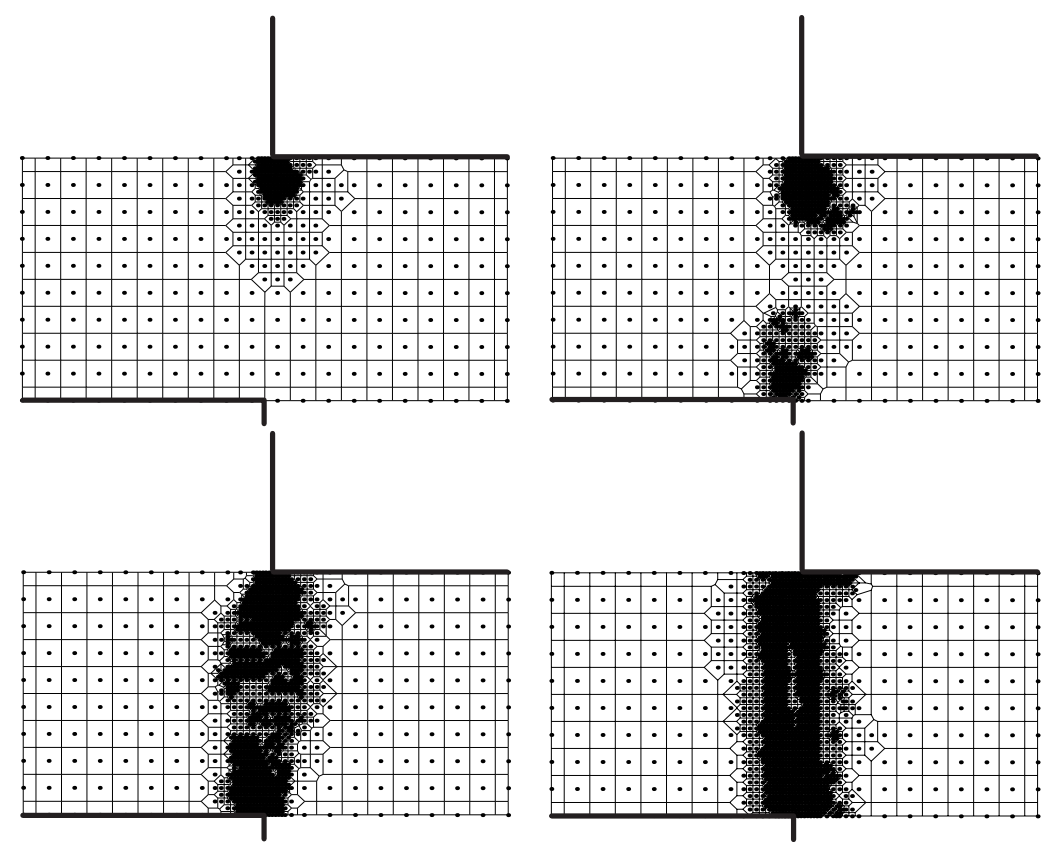

Figure 21. High-speed shearing $\left(R_{1}=0 \mathrm{~mm}\right.$ and $\left.R_{2}=0 \mathrm{~mm}\right)$ : adaptive refinement during shear band formation and constrained Voronoi cells

\section{CONCLUDING REMARKS}

Numerical simulation is currently a key issue for the development of efficient cutting processes. However, at the macroscopic scale or at the mesoscopic scale, one of the principal difficulties remains to have sufficiently representative mechanical models. With the macroscopic scale, these models are the cutting law with discontinuous cutting condition (case of milling) and dynamic models of the machine, in particular of the spindle. For the latter, and in particular for the spindles at high speed $(\omega>10000 \mathrm{rev} / \mathrm{min})$, the behavior is strongly nonlinear (bending according to the applied effort and the revolution speed). With the mesoscopic scale the definition and the identification of the constitutive laws at high speed and high temperature remain a major difficulty.

One way to solve some of these difficulties is to couple analysis at the macroscopic scale and at the mesoscopic scale. Indeed, the definition of consistent ways to go from one scale to another is of particular interest. For example if, at the macroscopic scale, thermal effect cannot be taken into account with detail near the tool tip(s) it can still be taken into acount for macroscopic deformations due to thermoelastic effects inside the workpiece. Thus, analysis done at the mesoscopic scale may give some help to construct cutting laws for the macroscopic scale with thermal aspects. A contrario, analysis done at the macroscopic scale can give various types of cutting condition to be simulated at the mesoscopic scale. Finally, many experimental data are obtained at a macroscopic level and then, the coupling of the two scales via an inverse approach will permit to achieve more representative models of metal cutting.

\section{ACKNOWLEDGEMENT}

At the mesoscopic scale this research was supported by the Cetim (French Industrial and Mechanical Technical Centre), and it is gratefully acknowledged. 

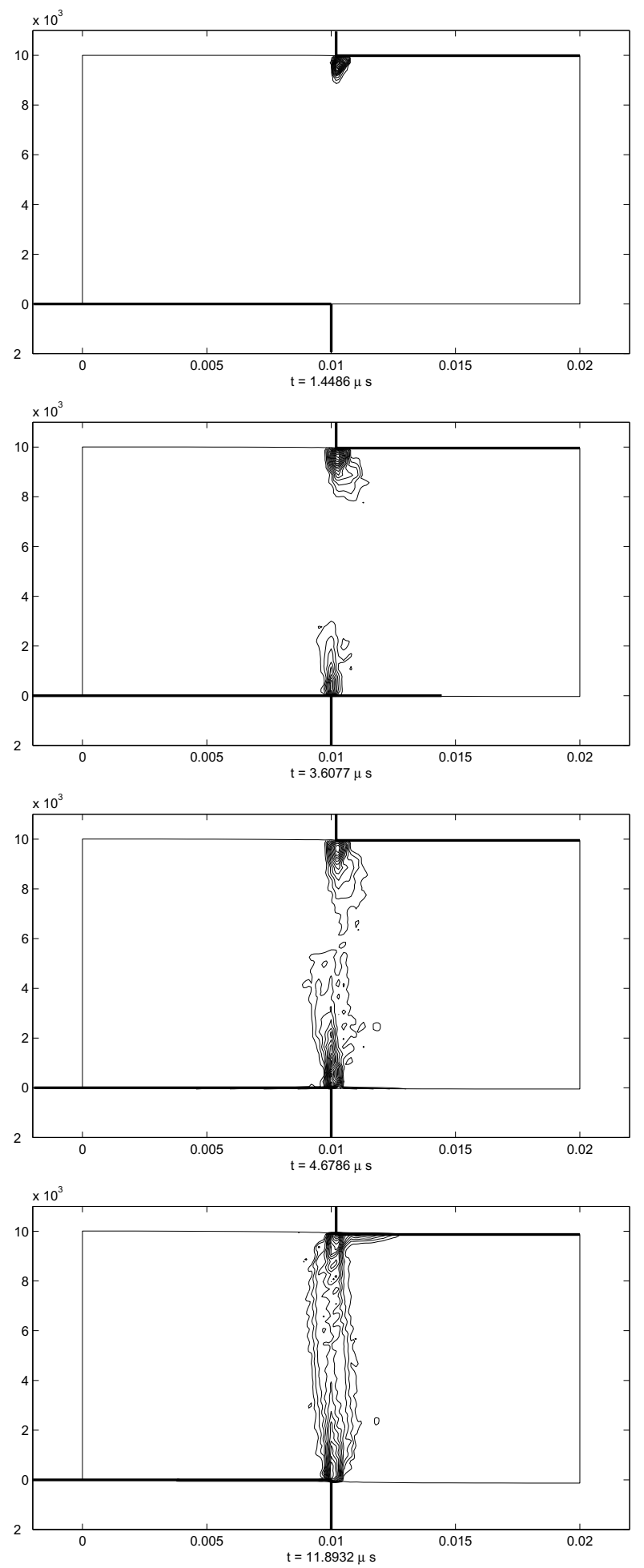

Figure 22. High-speed shearing $\left(R_{1}=0 \mathrm{~mm}\right.$ and $\left.R_{2}=0 \mathrm{~mm}\right)$ : shear band progression (equivalent plastic strain) 


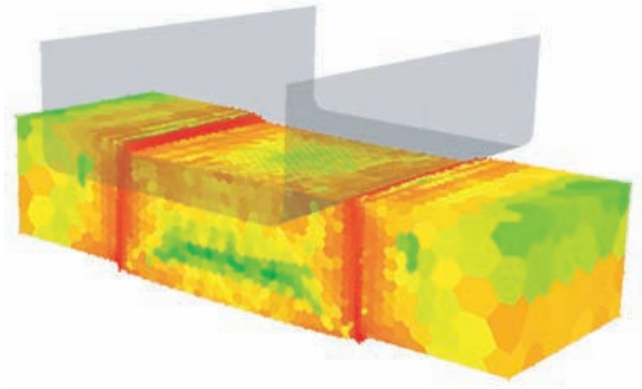

Mises equivalent stress

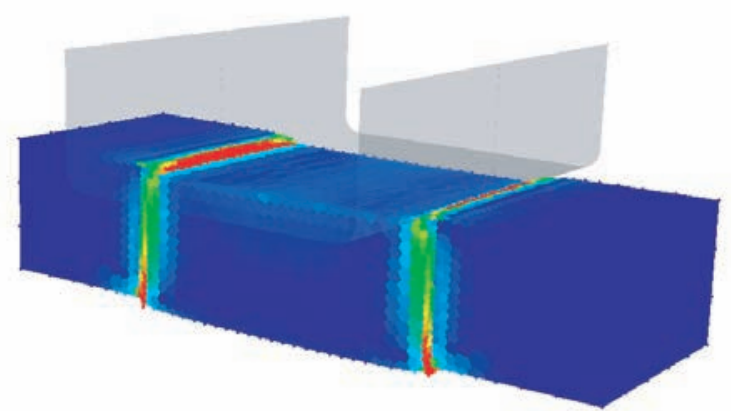

Plastic equivalent strain

Figure 23. 3D C-Nem simulation of blanking
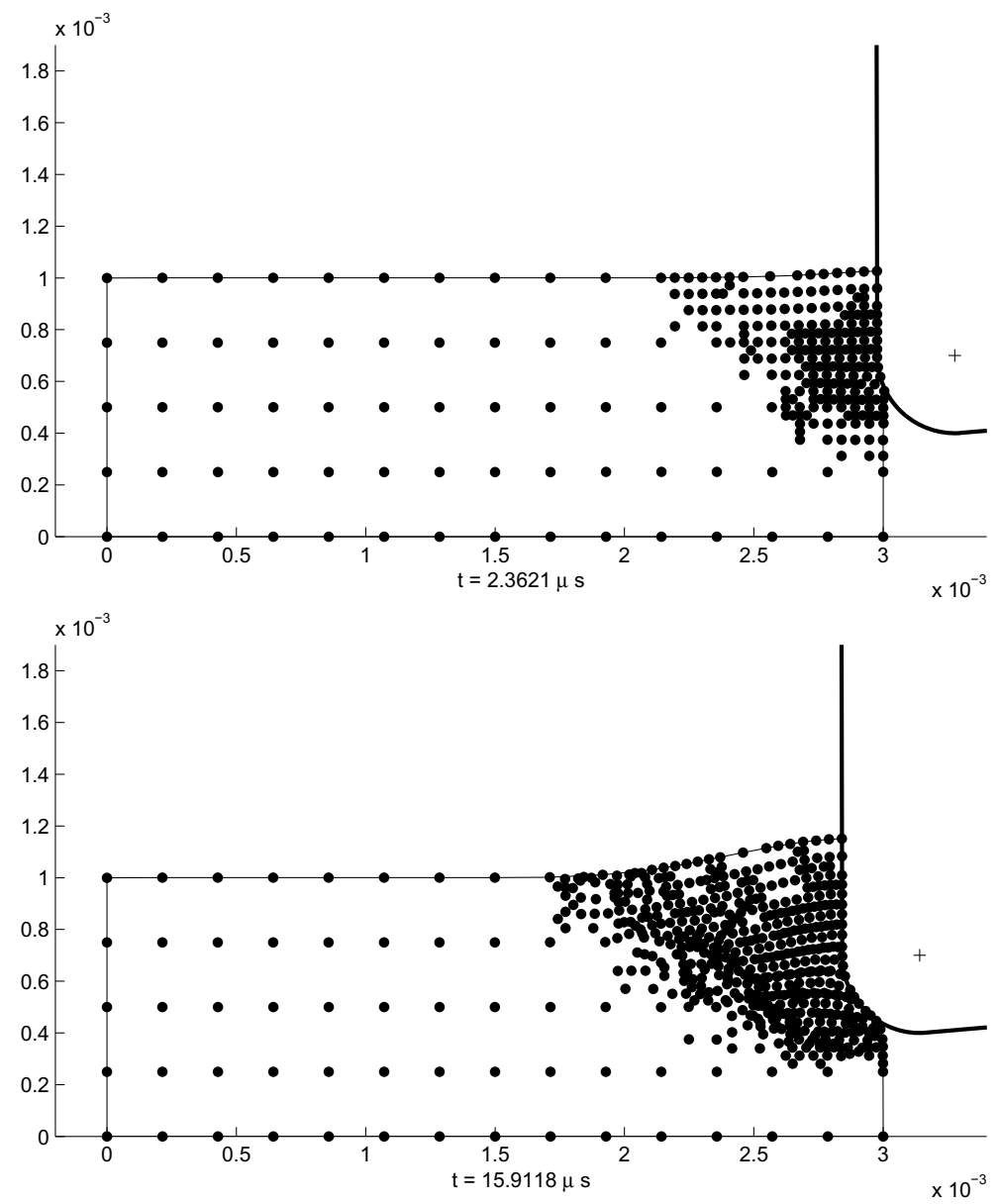

Figure 24. Adaptive refinement during cutting: shear localization 


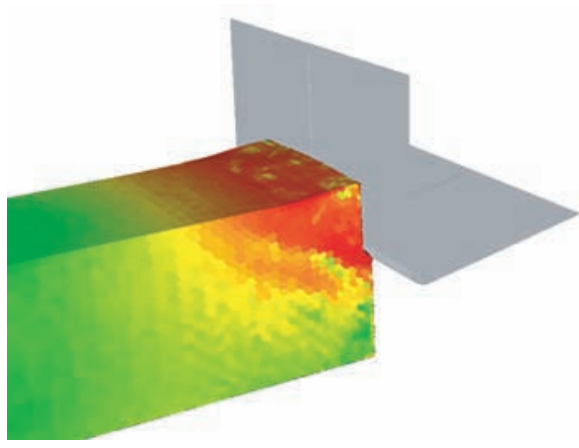

Mises equivalent stress

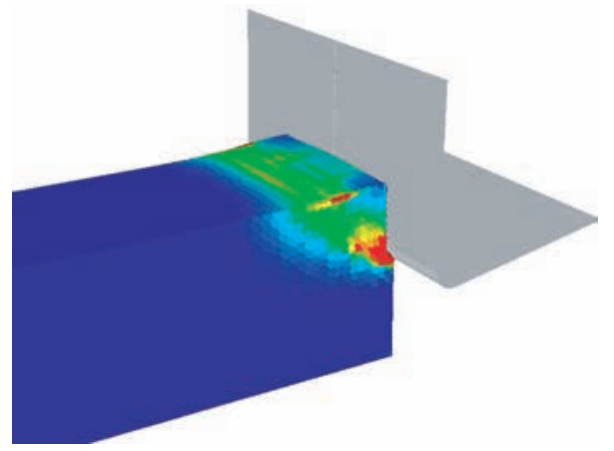

Plastic equivalent strain

Figure 25. 3D simulation of orthogonal cutting

\section{REFERENCES}

1 http://www.malinc.com/cutpro/.

2 Advantedge ${ }^{T M}$.

3 M. Touratier (1998). Computational models of chip formation in machining in a multi-scale approach. Present status and future needs. Technical Report, LM²S - Report, ENSAM Paris.

4 S. Jayarama, S. Kapoor and R.E. DeVor (2001). Estimation of the specific cutting pressures for mechanistic cutting force models. Int. J. Machine Tools, and Manufacture, 41(2), 265-281.

5 F. Atabey, I. Lazoglu and Y. Altintas. Mechanics of boring processes - Part I (2003). Int. J. Machine Tools and Manufacture, 43(5), 463-476.

6 H.E. Merritt (1965). Theory of self-excited machine tool chatter - resarch i. J. Engineering for Industry, 17, 447-454.

7 F. Ismail and E. Soliman (1997). A new method for the identification of stability lobes in machining. Int. J. Machine Tools, and Manufacture, 37(6), 763-774.

8 F. Abrari, M.A. Elbestawi and A.D. Spence (1998). On the dynamics of ball end milling: modeling of cutting forces and stability analysis. Int. J. Machine Tools, and Manufacture, $\mathbf{3 8}$, 215-237.

9 E. Budak and Y. Altintas (1998). Analytical prediction of chatter stability in milling-parti: General formulation. ASME Trans.- J. of Dynamic Systems, Measurement and Control, 120, 22-30, March.

10 E. Budak and Y. Altintas (1998. Analytical prediction of chatter stability in milling-partii: Application of the general formulation to common milling systems. ASME Trans.- J. of Dynamic Systems, Measurement and Control, 120, 31-36, March.

11 M.P. Volger, R.E. DeVor and S.G. Kapoor (2001). Nonlinear influence of effective lead angle in turning process stability. J. of Manufacturing Science and Engineering, 124, 473-475.

12 Y. Altintas, S. Engin and E. Budak (1999). Analytical stability prediction and design of variable pitch cutters. J. of Manufacturing Science and Engineering, 121, 173-178, May.

13 F. Lapujoulade (2003). Simulation des phénomènes vibratoires intervenant en usinage. $\mathrm{PhD}$ thesis, INSA Lyon et Université Claude Bernard Lyon I.

14 S. Engin and Y. Altintas (2001). Mechanics and dynamics of general milling cutters. Part I: helical end mills. Int. J. Machine Tools, and Manufacture, 41, 2195-2212.

15 S. Engin and Y.Altintas (2001). Mechanics and dynamics of general milling cutters. Part II: inserted cutters. Int. J. Machine Tools, and Manufacture, 41, 2213-2231. 
16 A. Grefioz (2000). Calcul par ééments finis d'un banc d'essai. In Premières Assises Machines et Usinage à Grande Vitesse, March.

17 Y. Altintas (2000). Modelling approaches and software for predicting the performance of milling operations at mal-ubc. In CIRP 2000 Machining Workshop.

18 J. Gradišek, M. Kalveram, T. Insperger, K. Weinert, G. Stépán, E. Govekar and I. Grabec (2004). On stability prediction for low radial immersion milling. In A. Moisan and G. Poulachon (Eds.), $7^{\text {th }}$ CIRP International Workshop on Modeling of Machining Operations, pp. 227-233.

19 D. Montgomery and Y. Altintas (1991). Mechanism of cutting force and surface generation in dynamic milling. J. of Engineering for Industry, 113, 160-168, May.

20 G. Coffignal, E. Beauchesne, K. Dekelba and N. Hakem (1996). Mechanical simulation of machining using cutting tools. In 1st International Conference - IDMME'96, Volume 1, pp. $145-154$.

21 A. Marty, P. Lorong and G. Coffignal (2000). Numerical simulation of a turning operation. In II International Seminar on Improving Machine Tool Performance, La Baule de Nantes de France, July.

22 S. Assouline, E. Beauchesne, G. Coffignal, P. Lorong and A. Marty (2002). Simulation numérique de l'usinage à l'échelle macroscopique : modèles dynamiques de la pièce. Mécanique et Industrie, 3, 389-402.

23 F. Lapujoulade, T. Mabrouki, and K. Raissi (2002). Prédiction du comportement vibratoire du fraisage latéral de finition des piéces à parois minces. Mécanique et Industries, 3, 403-418.

24 T. Van Hook (1986). Real-time shaded nc milling display. In SIGGRAPH'86, pp. 15-35.

25 G.M. Kim, P.J. Cho and C.N. Chu (2000). Cutting force prediction for scultured surface ball-end milling using z-map. Int. J. Machine Tools, and Manufacture, 40, 277-291.

26 Y. Mizugaki, M. Hao and K. Kikkawa (2001). Geometric generating mechanism of machined surface by ball-nosed end milling. In Annals of the CIRP, Volume 50.

27 S. Ratchev, S. Liu, W. Huang and A.A. Becker (2004). Milling error prediction and compensation in machining of low-rigidity parts. Int. J. Machine Tools, and Manufacture, 44, 1629-1641.

28 K. Weinert and A. Zabel (2004). Simulation based tool wear prediction in milling of sculptured surfaces. Annals of the CIRP, 53, 217-223.

29 W.M.M. Ng and S.T. Tan (2000). Incremental tessellation of trimmed parametric surfaces. Computer Aided Design, 32(4), 279-294.

30 R. Radulescu, S.G. Kapoor and R.E. DeVor (1993). An investigation of variable spindle speed face milling for tool-work structures with complex dynamics: Part 1- simulation results. J. of Manufacturing Science and Engineering, 64, 603-614.

31 R. Radulescu, S.G. Kapoor and R.E. DeVor (1993). An investigation of variable spindle speed face milling for tool-work structures with complex dynamics: Part 2- physical explanation. $J$. of Manufacturing Science and Engineering, 64, 615-627.

32 Khaled Dekelbab (1995). Modélisation et simulation du comportement dynamique de l'ensemble Pièce-Outil-Machine en usinage par outil coupant. PhD thesis, Ecole Nationale Supérieure d'Arts et Métiers - CER de Paris.

33 K. Mehdi, J.-F. Rigal and D. Play (2002). Dynamic behavior of a thin-walled cylindrical workpiece during the turning process, Part 2: experimental approach and validation. J. of Manufacturing Science and Engineering, 124, 569-580.

34 B.K. Choi and R.B. Jerard (1998). Sculptured surface machining. Kluwer Academic Publishers.

35 K.J. Bathe (1996). Finite Element Procedures. Prentice-Hall.

36 M. Géradin and D. Rixen (1996) . Théorie des vibrations. Application à la dynamique des structures. Masson. 
37 S.P.F.C. Jaspers and J.H. Dautzenberg (2002). Material behaviour in metal cuttingl: strains, stain rates and temperatures in chip formation. J. Material Processing Technology, 121, 123135.

38 J.-D. Kim and C.-H. Moon (1996). A study on microcutting for the configuration of tools using molecular dynamics. J. Material Processing Technology, 59, 309-314.

39 R.G. Rentsch (2004). Molecular dynamics simulation of microscopic phenomena in cutting and forming processes. In ESAFORM, pp. 93-96, Trondheim, Norway, April 28-30.

40 H. Hernst (1938). Physics of metal cutting. Machining of Metals - American Society for Metals, page 24 .

41 H. Hernst and M.E. Merchant (1941). Chip formation, friction and high quality machined surfaces. Trans. American Society for Metals, 29, 299-378.

42 M.E. Merchant (1945). Mechanics of the metal cutting process: I. Orthogonale cutting and type 2 chip. Journal of Applied Physics, 16, 267-275.

43 N. Fang, I.S. Jawahir and P.L.B. Oxley (2001). A universal slip-line model with non-unique solutions for machining with curled chip formation and a restricted contact tool. Int. J. Mech. Sci., 43, 557-580.

44 A. Molinari and A. Moufki (2005). A new thermomechanical model of cutting applied to turning operations. part i. theory. Int. J. Machine Tools, and Manufacture, 45, 166-180.

45 A. Moufki and A. Molinari (2005). A new thermomechanical model of cutting applied to turning operations. part ii. parametric study. Int. J. Machine Tools, and Manufacture, 45, $181-193$.

46 A. Moufki, A. Devillez, D. Dudzinski and A. Molinari (2004). Thermomechanical modelling of oblique cutting and experimental validation. Int. J. Machine Tools, and Manufacture, 44, 971-989.

47 Y. K. Chou and H. Song (2005). Thermal modeling for white layer predictions in finish hard turning. Int. J. Machine Tools, and Manufacture, 45, 481-495.

48 S.-L. Ko and A. Dornfeld (1996). Burr formation and fracture in oblique cutting. J. of Material Processing Technology, 62, 24-36.

49 V.R. Marinov (2001). Hybrid analytical-numerical solution for the shear angle in orthogonal metal cutting - part i: theoretical foundation. Int. J. Mech. Sci., 43, 399-414.

50 K. Okushima and Y. Kakino (1971). The residual stress produced by metal cutting. Annals of the CIRP, 20(1), 13.

51 A.O. Tay, M.G. Stevenson and G.D. Davis (1974). Using finite element method to determine the temperature distribution in orthogonal in orthogonal machining. Proceedings of Institute of Mechanical Enginners, 188, 627.

52 M.G. Stevenson, P.K. Wright and J.G. Chow (1983). Further developments in applying finite element technique to the calculation of temperature distribution in machining and compressions with experiment. ASME Journal of Engineering for Industry, 105, 149-154.

53 R. Natarajan and S. Jeelani (1983). Residual stresses in machining using finite element method. Computer in Engineering, Computer Software Applications, 3, 79-80.

54 K.F. Ehmann, S.G. Kapoor, R.E. DeVor and I. Lazoglu (1997). Machining process modeling: A review. J. of Manufacturing Science and Engineering, 119, 655-663.

55 J. Mackerle (1999). Finite-element analysis and simulation of machining: a bibliography (19761996). J. of Material Processing Technology, 86, 17-44.

56 G.R. Johnson and W.H. Cook (1983). A constitutive model and data for metals subjected to large strains, high strain rates and high temperatures. In Proceeding of the $7^{\text {th }}$ International Symposium on Ballistics, pp. 541-547, The Hague, Holland, April. 
57 T. Ozel and E. Zeren (2004). Determination of material work flow stress and friction for fef of machining using orthogonal cutting tests. J. Proc. Tec., 153-154, 1019-1025.

58 D. Steinberg, S. Cochram and M. Guinan (1980). A constitutive model for metals applicable at high-strain rate. J. of Applied Physics, 51(3), 1498-1504.

59 W.K. Rule and S. Jones (1998). A revised form for the johnson-cook strength model. Int. J. of Impact Engng, 21(8), 609-624.

60 G.R. Johnson and W.H. Cook (1985). Fracture caracteristics of three metals subjected to various strains, strain rates, temperatures and pressure. Engineering Fracture Mechanics, $\mathbf{2 1}(1), 31-48$.

61 Z.-C. Lin and S.Y. Lin (1992). A coupled finite element model thermo-elastic-plastic large deformation for orthogonal cutting. J. of Engineering Mater. and Technol., 114, 218-226.

62 T. Shirakashi, K. Maekawa and E. Usui (1993). Flow stress of low carbon steel and high temperature and strain rate. J. of Jap. Society for Production Engng, 17, 167-172.

63 Z.-C. Lin and Y.-Y. Lin (1999). Fundamental modeling for oblique cutting by thermo-elasticplastic fem. Int. J. Mech. Sci., 41, 941-965.

64 A.G. Mamalis, M. Horváth, A.S. Branis and D.E. Manolakos (2001). Finite element simulation of chip formation in othogonal metal cutting. J. of Material Processing Technology, 110, 12-27.

65 Y.-C. Yen, A. Jain and T. Altan (2004). A finite element analysis of orthogonal machining using different tool edge geometries. J. of Material Processing Technology, 146, 72-81.

66 J. S. Wu, O. W. Dillon and W. Y. Lu (1996). Thermo-viscoplastic modeling of machining process using a mixed finite element method. J. of Manufacturing Science and Engineering, 118, 470-482.

67 D.-C. Ko, S.-L. Ko and B.-M. Kim (2002). Rigid-thermoviscoplastic finite element simulation of non-steady-state orthogonal cutting. J. of Material Processing Technology, 130-131, 345-350.

68 J. Lemonds and A. Needleman (1986). Finite element analysis of shear localization in rate and temperature dependent solids. Mechanics of Materials, 5, 339-361.

69 T.D. Marusich and M. Ortiz (1995). Modelling and simulation of high-speed machining. Int. J. Numer. Meth. in Engng, 38, 3675-3694.

70 S. Lei, Y.C. Shin and F.P. Incorpera (1997). Material constitutive modeling under high strain rates and temperatures through orthogonal machining tests. In Proceedings of Manufacturing Science and Engineering, ASME IMECE, MED, Volume 6-2, pp. 91-98.

71 S. Lei, Y.C. Shin and F.P. Incorpera (1999). Thermo-mechanical modelling of orthogonal machining process by finite element analysis. Int. J. Machine Tools, and Manufacture, 39(15), 731-750, May.

72 J. Söder and T. Altan (2001). Material database for manufacturing simulation (madams): Summary of the activities and flow stress database. ERC for Net Shape Manufactureing HPM/ERC/NSM-01-R-76, Ohio State University.

73 Firas Ali (2001). Modélisation et simulation thermomécaniques de la coupe des métaux. PhD thesis, Ecole Nationale Supérieure d'Arts et Métiers - CER de Paris, Juillet.

74 J. S. Strenkowski and J. T. Carroll (1986). An orthogonal metal cutting model based on an eulerian finite element method. In Manufacturing Processes, Machines and Systems, Proceedings of the $13^{\text {th }}$ NSF Conference on Production Research an Technology.

75 D. J. Benson and S. Okazawa (2004). Simulations of high-speed machining using a multimaterial finite element formulation. In S. Ghosh, J.C. Castro, and J.K. Lee (Eds.), NUMIFORM, Material Processing and Design: Modeling, Simulation and Applications, pp. 13411346 .

76 J.T. Carroll and J.S. Strenkowski (1988). Finite element models of orthogonal cutting with application to single point diamond turning. Int. J. Mech. Sci., 30, 899-920. 
77 R.A. Riddle (1987). The effect of the failure criterion in the numerical modeling of orthogonal metal cutting. Technical Report UCRL-93745, Lawrence Livermore Report.

78 H.T.Y. Yang, M. Heinstein and A.J.M. Shih (1989). Adaptative 2d finite element simulation of metal forming processes. Int. J. Numer. Meth. in Engng, 28, 1409-1428.

79 K. Komvopoulos and S. A. Erpenbeck (1991). Finite element modeling of orthogonal metal cutting. ASME Journal of Engineering for Industry, 113, 253-267.

80 S.F. Wayne, C. Simmerman and D.A. Oniel (1993). Aspects of advanced cutting tool and chip-flow modeling. In H. Bildstein and R. Eck, editors, Proceedings of the $13^{\text {th }}$ International Plansee Seminar, Volume 2, page 64. Metalwerk Plansee, Reutte.

81 A. J. Shih (1996). Finite element analysis of orthogonal metal cutting mechanics. Int. J. Machine Tools, and Manufacture, 36(2), 255-273.

82 M. Baker, J. Rosler, and C. Siemers (2002). A finite element model of high speed metal cutting with adiabatic shearing. Comp. and Struc., 80, 495-513.

83 H. Sasahara, T. Obikawa and T. Shirakashi (2004). Prediction model of surface residual stress within a machined surface by combining two orthogonal plane models. Int. J. Machine Tools, and Manufacture, 44, 815-822.

84 L.-J. Xie, J. Schmidt, C. Schmidt and F. Biesinger (2005). 2d fem estimate of tool wear in turning operation. Wear, 258, 1479-1490.

85 G. Camacho, T. Marusich and M. Ortiz (1996). Adaptative meshing methods for the analysis of unconstrained plastic flow. Advanced Computational Methods for Material Modeling, 268, $71-83$.

86 E. Ceretti, M. Lucchi and T. Altan (1999). FEM simulation of orthogonal cutting: serrated chip formation. J. of Material Processing Technology, 95:17-26.

87 Y. B. Guo and D. W. Yen (2004). A fem study on mechanisms of discontinuous chip formation in hard machining. J. of Material Processing Technology, 155-156, 1350-1356.

88 O. Pantalé, J.-L. Bacaria, O. Dalverny, R. Rakotomalala and S. Caperaa (2004). 2d and 3d numerical models of metal cutting with damage effects. Comp. Meth. Applied Mech. and Eng., 193, 4383-4399.

89 M. Barge, H. Hamdi, J. Rech and J-M. Bergheau (2005). Numerical modelling of orthogonal cutting: influence of numerical parameters. J. of Material Processing Technology. in press.

90 Deform 3d user manual, 1997.

91 E. Ceretti, C. Lazzaroni, L. Menegardo and T. Altan (2000). Turning simulations using a three-dimensional FEM code. J. of Material Processing Technology, 98, 99-103.

92 R. Rokotomalala, P. Joyot and M. Touratier (1993). Arbitrary lagrangian-eulerian thermomechanical finite element model of material cutting. Communication in Numerical Methods in Engineering, 9, 975-987.

93 L. Olovsson, L. Nilsson and K. Simonsson (1999). An ALE formulation for the solution of two-dimensional metal cutting problems. Comp. and Struc., 72, 497-507.

94 M. Baker (2004). Finite element investigation of the flow stress dependence of chip formation. J. of Material Processing Technology.

95 M. Baker, J. Rosler and C. Siemers (2003). The influence of thermal conductivity on segmented chip formation. Computational Materials Science, 26, 175-182.

96 ABAQUS. Abaqus/Explicit User's Manual. HKS Inc., 1997.

97 ABAQUS. ABAQUS/Standard User's Manual. HKS Inc., 1997.

98 LS-Dyna. Ls-dyna theorical Manual. L.S.T. Corporation, California, USA, 1997. 
99 Halil Gil, S. Engin Engin Kiliç and A. Erman Tekkaya (2004). A comparison of orthogonal cutting data from experiments with three different finite element models. Int. J. Machine Tools, and Manufacture, 44, 933-944.

100 L. Fratini, A. Lombardo and F. Micari (1996). Material characterization for the prediction of ductile fracture occurrence: an inverse approach. Journal of Materials Processing Technology, 60, 311-316.

101 E.H. Lee (1969). Elastic-plastic deformation at finite strains. J. Applied Mech. ASME, 36, $1-6$.

102 K.J. Bathe (1986). Finite Element procedures. Prentice Hall.

103 D. Perić, M. Vaz and D.R.J. Owen (1999). On adaptive strategies for large deformations of elasto-plastic solids at finite strains: computational issues and industrial applications. Comp. Meth. Applied Mech. and Eng., 176, 279-312.

104 A.L. Eterovic and K.-J. Bathe (1990). A hyperelastic-based large strain elasto-plasstic constitutive formulation with combined isotropic-kiematic hardening using the logarithmic stress and strain measures. Int. J. Numer. Meth. in Engng, 30, 1099-1114.

105 J.C. Simo (1992). Algorithms for static and dynamic multiplicative plasticity that preserve the classical return-mapping algorithm schemes of the infinitesimal theory. Comp. Meth. Applied Mech. and Eng., 99, 61-112.

106 A. Cuiti no and M. Ortiz (1992). A material-independent method for extending stress-update algorithms from small-strain plasticity to finite plasticity with multiplicative kinematics. Engineering computations, 9, 437-451.

107 J.C. Simo and C. Miehe (1992). Associative coupled thermoplasticity at finite strains : formulation numerical analysis and implementation. Comp. Meth. Applied Mech. and Eng., 98, 41-104.

108 J.C. Simo and T.J.R. Hughes (1998). Computational inelasticity. Springer-Verlag.

109 J.S. Chen, Y. Yoon and C.T. Wu (2002). Non-linear version of stabilized conforming nodal integration for galerkin mesh-free methods. Int. J. Numer. Meth. Engng, 53, 2587-2615.

110 G. Taylor and H. Quinney (1934). The latent energy remaining in a metal after cold working. Proceedings of the Royal Society, 143, 307-326.

111 B. Nayroles, G. Touzot and P. Villon (1992). Generalizing the finite element method: diffuse approximation and diffuse elements. Computational mechanics, 10, 307-318.

112 T. Belytschko, Y.Y. Lu and L. Gu (1994). Element-free galerkin methods. Int. J. Numer. Meth. in Engng, 37, 229-256.

113 W.K. Liu, S. Jun and Y.F. Zhang (1995). Reproducing kernel particle methods. Int. J. Numer. Methods Fluids., 21, 1081-1106.

114 L.B. Lucy (1977). A numerical approach to the testing of fuion process. The astronomical journal, 88, 1013-1024.

115 S. Gupta M. Sharan and E.J. Kansa (1997). Application of the multiquadratic method for numerical solution of elliptic partial differential equations. Applied Mathematics and Computation, 84, 837-857.

116 N. Sukumar, B. Moran and T. Belytschko (1998). The natural elements method in solid mechanics. Int. J. Numer. Meth. in Engng, 43, 839-887.

117 S. Li, W. Hoa and W.K. Liu (2000). Mesh-free simulations of shear banding in large deformation. Int. Journ. Solids. and Sruct., 37, 7183-7206.

118 I. Alfaro, D. Gonzalez, D. Bel, E. Cueto, M. Doblare and F. Chinesta (2004). Recent advances in the meshless simulation of aluminium extrusion and other related forming processes. Archives of Computational Methods in Engineering. to appear. 
119 J. Yvonnet, P. Lorong, D. Ryckelynck and F. Chinesta (2005). Simulating dynamic thermoelastoplasticity in large transformations witha adaptive refinement in the natural element method: application to shear banding. Int. J. Forming Processes. accepted.

120 J.S. Chen, C. Pan and C.T. Wu (1997). Large deformation analysis of rubber based on a reproducing kernel particle method. Computer methods in applied mechanics and enginnering, 173, 99-109.

121 J. Dolbow and T. Belytschko (1999). Volumetric locking in the element-free galerkin method. Int. J. Numer. Meth. in Engng, 46, 925-942.

122 W.K. Liu, S. Li and T. Belytschko (1997). Moving least square reproducing kernel method (i) methodology and convergence. reproducing kernel particle methods. Comput. Meth. Appl. Mech. Engng., 143, 113-154.

123 P. Krysl and T. Belytschko (1996). Element-free galerkin : convergence of the continuous and discontinuous shape functions. Comp. Meth. Applied Mech. and Eng..

124 J. Yvonnet, D. Ryckelynck, P. Lorong and F. Chinesta (2004). A new extension of the natural element method for non convex and discontinuous domains: the constrained natural element method (c-nem). Int. J. Numer. Meth. in Engng, 60, 1451-1474.

125 M. Sambridge, J. Braun and H. McQueen (1995). Geophysical parameterization and interpolation of irregular data using natural neighbours. Geophys. J. Int., 122, 837-857.

126 R. Sibson (1980). A vector identity for the dirichlet tesselations. Math. Proc. Camb. Phil. Soc., 87, 151-155.

127 H. Hiyoshi and K. Sugihara (2002). Improving continuity of voronoi-based interpolation over delaunay spheres. Computational Geometry, 22, 167-183.

128 V.V. Belikov, V.D. Ivanov, V.K. Kontorovich, S.A. Korytnik and A.Y. Semenov (1997). The non-sibsonian interpolation : a new method of interpolation of the values of a function on an arbitrary set of points. Computational Mathematics and Mathematical Physics, 37(1), 9-15.

129 E. Cueto, M. Doblaré and L. Gracia (2000). Imposing essential boundary conditions in the natural elements method by means of density-scaled alpha-shapes. Int. J. Numer. Meth. in Engng, 49, 519-546.

130 R. Seidel (1988). Constrained delaunay triangulations and voronoi diagrams with obstacles. In 1978-1988 Ten Years IIG, pp. 178-191.

131 E. Schönhardt (1928). Uber die zerlegung von dreieckspolyedern in tetraeder. Math. Annalen, 98.

132 JR Shewchuck (1998). Tetrahedral mesh generation by delaunay refinement. In Proceedings of the fourteenth annual symposium on computational geometry, Minneapolis, Minnesota, pp. 86-95, June. Association for Computing Machinery.

133 JR Shewchuck (2000). Sweep algorithms for constructing higher-dimensional constrained delaunay triangulations. In Proceedings of the sixteenth annual symposium on computational geometry, pp. 350-359, June. Association for Computing Machinery.

134 J. Yvonnet, F. Chinesta, P. Lorong and D. Rynckelynck (2005). The constrained natural element method (c-nem) for treating thermal models involving moving interfaces. International Journal of Thermal Sciences, 44, 559-569.

135 J. Lemaitre and J. L. Chaboche (1990). Mechanics of solid materials. Cambridge University Press, UK.

136 J.-F. Yau, S.-S. Wang and H.T. Corten (1980). A mixed-moed crack analysis of isotropic solids using conservation laws of elasticity. J. Applied Mech. ASME, 47, 335-341.

137 J.M. Melenk and I. Babuška (1996). The partition of unity finite element method : basic theory and applications. Comp. Meth. Applied Mech. and Eng., 139, 289-314.

138 O.C. Zienkiewicz and J.Z. Zhu (1987). A simple error estimator and adaptive procedure for practical engineering analysis. Int. J. Numer. Meth. in Engng, 24, 337-357. 
139 N.-S. Lee and K.J. Bathe (1994). Error indicators and adaptive remeshing in large deformation finite element analysis. Finite Element Analysis Design, 16, 99-139.

140 G.T. Camacho and M. Ortiz (1996). Computational modeling of impact damage in brittle materials. Int. J. Solids Struc., 33, 2899-2938.

141 P. Ladevèze (1998). Non Linear Computational Structural Mechanics. Springer.

142 I. Babuška and W.C. Rheinboldt (1979). Adaptive approaches and reliability estimations in finite element analysis. Comp. Meth. Applied Mech. and Eng., 17/18, 519-40.

143 P. Ladevèzeand G. Coffignal and J.P. Pelle (1998). Accuracy of elastoplastic and dynamic analysis. In Babuška et al. eds., Accuracy estimates and adaptive refinements in finite element computations, pp. 86-95. John Wiley, June. Association for Computing Machinery.

144 T. Belytschko, B.L. Wong and E.J. Plakacz (1989). Fission-fusion adaptivity in finite elements for nonlinear dynamics of shells. Comp. and Struc., 33, 1307-1323.

145 M. Ortiz and J.J. Quigley (1991). Adaptive mesh refinement in strain localization problems. Comp. Meth. Applied Mech. and Eng., 90, 781-804.

146 L. Gallimard, P. Ladevèze and J.P. Pelle (1996). Error estimation and adaptivity in elastoplasticity. Int. J. Numer. Meth. in Engng, 39, 189-217.

147 W.K. Liu, R.A. Uras and Y. Chen (1997). Enrichment of the finite element method with the reproducing kernel particle method. J. Applied Mech. ASME, 64, 861-870.

148 Y. You, J.S. Chen and H. Lu (2003). Filters, reproducing kernel, and adaptive meshfree method. Computational mechanics, 31, 316-326.

149 H-J. Chung and T. Belytschko (1998). An error estimate in the efg method. Computational Mechanics, 21, 91-100.

150 C.K. Lee and C.E. Zhou (2003). On error estimation and adaptive refinement for element free Galerkin method. Part I: stress recovery and a posteriori error estimation. Comp. ES Struc., 82(4-5), 413-428.

151 C.K. Lee and C.E. Zhou (2003). On error estimation and adaptive refinement for element free Galerkin method. Part II: adaptive refinement. Comp. ES Struc., 82(4-5), 429-443.

152 H. Lu and J.S. Chen (2002). Adaptive meshfree particle method. Lecture notes in Computational Science and Engineering, 26, 251-267.

153 J. Yoo, B. Moran and J.-S. Chen (1998). Stabilized conforming nodal integration in the naturalelement method. Int. J. Numer. Meth. in Engng, 60, 861-890. 